\title{
Optical novae: the major class of supersoft X-ray sources in $\mathrm{M} 31^{\star}$
}

\author{
W. Pietsch ${ }^{1}$, J. Fliri ${ }^{2, \star \star}$, M. J. Freyberg ${ }^{1}$, J. Greiner ${ }^{1}$, F. Haberl ${ }^{1}$, A. Riffeser ${ }^{1,2, \star \star}$, and G. Sala ${ }^{1,3}$ \\ 1 Max-Planck-Institut für extraterrestrische Physik, Giessenbachstraße, 85741 Garching, Germany \\ e-mail: wnp@mpe.mpg.de \\ 2 Universitätssternwarte München, Scheinerstraße, 81679 München, Germany \\ ${ }^{3}$ Institut d'Estudis Espacials de Catalunya (ICE-CSIC), Campus UAB, Facultat de Ciencies, 08193 Bellaterra, Spain
}

Received 24 March 2005 / Accepted 19 June 2005

\section{ABSTRACT}

\begin{abstract}
We searched for X-ray counterparts of optical novae detected in M31 and M33. We combined an optical nova catalogue from the WeCAPP survey with optical novae reported in the literature and correlated them with the most recent X-ray catalogues from ROSAT, XMM-Newton, and Chandra, and - in addition - searched for nova correlations in archival data. We report $21 \mathrm{X}$-ray counterparts for novae in M 31 - mostly identified as supersoft sources (SSS) by their hardness ratios - and two in M33. Our sample more than triples the number of known optical novae with a supersoft X-ray phase. Most of the counterparts are covered in several observations allowing us to constrain their X-ray light curves. Selected brighter sources were classified by their XMM-Newton EPIC spectra. We use the well-determined start time of the SSS state in two novae to estimate the hydrogen mass ejected in the outburst to $\sim 10^{-5} M_{\odot}$ and $\sim 10^{-6} M_{\odot}$, respectively. The supersoft X-ray phase of at least $15 \%$ of the novae starts within a year. At least one of the novae shows a SSS state lasting 6.1 years after the optical outburst. Six of the SSSs turned on between 3 and 9 years after the optical discovery of the outburst and may be interpreted as recurrent novae. If confirmed, the detection of a delayed SSS phase turn-on may be used as a new method to classify novae as recurrent. At the moment, the new method yields a ratio of recurrent novae to classical novae of 0.3 , which is in agreement (within the errors) with previous works.
\end{abstract}

Key words. galaxies: individual: M 31 - galaxies: individual: M 33 - stars: novae, cataclysmic variables $\mathrm{X}$-rays: galaxies $-\mathrm{X}$-rays: binaries

\section{Introduction}

After the first discovery of X-ray emission from nova GQ Mus 463 days after outburst (Ögelman et al. 1984) and its subsequent detection by ROSAT nine years after outburst (Ögelman et al. 1993), it was widely believed that post-novae should go through an extended phase of residual hydrogen burning and appear as a supersoft X-ray source (SSS). Combining the burning rate of $10^{-7} M_{\odot} / \mathrm{yr}$ with the assumption that there should be about $10^{-4} M_{\odot}$ in the shell to ignite hydrogen burning, a duration of the burning phase of $10^{3}$ to $10^{4} \mathrm{yr}$ has been predicted (MacDonald et al. 1985; Starrfield 1989). However, supersoft X-ray emission has been detected so far for only five other novae: V1974 Cyg detected as SSS for about 400 days starting from 250 days after outburst (Krautter et al. 1996);

* Partly based on observations with XMM-Newton, an ESA Science Mission with instruments and contributions directly funded by ESA Member States and NASA, and on observations obtained with the Wendelstein Observatory of the Universitätssternwarte München.

$\star \star$ Visiting astronomer at the German-Spanish Astronomical Center, Calar Alto, operated by the Max-Planck-Institut für Astronomie, Heidelberg, jointly with the Spanish National Commission for Astronomy.
Nova LMC 1995 was detected as SSS from 200 to more than 2100 days after outburst (Orio \& Greiner 1999; Orio et al. 2003), but had almost faded after 8 years (Orio 2004); V382 Vel, with an intense SSS component, detected about 180 and 222 days after outburst, but disappearing by day 268 (Orio et al. 2002; Burwitz et al. 2002); V1494 Aql about 250 days after outburst (Drake et al. 2003) and no later X-ray observations reported; V4743 Sgr about 180 days after outburst, and still appearing as SSS at day 740 after outburst (Ness et al. 2003; Orio \& Tepedelenlioglu 2004). In a systematic search through all ROSAT pointed observations covering 39 novae over a time span up to $10 \mathrm{yr}$, no further supersoft phase was discovered (Orio et al. 2001).

Although only X-ray observations can provide direct insight into the hot post-outburst white dwarf, ultraviolet emission lines arising from the ionization of the ejecta by the central X-ray source reflect the presence of on-going hydrogen burning on the white dwarf surface. Several works have used this indirect indicator to determine the turn-off of classical novae from IUE observations (Shore et al. 1996; Gonzalez-Riestra et al. 1998; Vanlandingham et al. 2001), showing in all cases turn-off times shorter than expected. The short duration of the 
H-burning phase derived from observations could be explained by a small post-outburst envelope mass, suggesting the presence of some extra mass loss mechanism acting after the nova outburst, i.e., a thick wind (Kato \& Hachisu 1994) or a common envelope (MacDonald et al. 1985). In fact, post-nova white dwarf envelopes with steady $\mathrm{H}$-burning are stable only for masses smaller than about $10^{-5} M_{\odot}$ (Tuchman \& Truran 1998; Sala \& Hernanz 2005a), which also suggests that instabilities in envelopes with larger masses could contribute to getting rid of the mass excess. With the low Galactic novae rate there was no substantial improvement in our understanding of these processes during the last $5 \mathrm{yr}$ with Chandra and XMM-Newton observations.

However, observing the nova population in M31 has the advantage that this, as well as several additional questions, can be attacked much more easily as compared to the local sources (including those in the Magellanic Clouds): (i) what is the spatial distribution over the galaxy, and are there possible correlations with different environment? (ii) What is the size of the population, including the fraction of sources detected during their supersoft X-ray emission phase? (iii) What is the variability pattern? (iv) Are there correlations between the optical and $\mathrm{X}$-ray properties?

There have been many surveys for optical novae in M31 starting with the early work of Hubble (1929), who used the novae to establish the distance of M 31, already estimated a yearly nova rate of 30 , and found that novae are most frequent in the nuclear area. Novae were detected by comparing plates taken at different times. However, many were missed due to the sparse sampling and the shortness of the nova outbursts. When it was noticed that novae stayed bright in $\mathrm{H} \alpha$ for a longer time, this band was used for M 31 nova searches (see e.g. Ciardullo et al. 1990). With this method many nova candidates were detected with only rough knowledge of the date of outburst, as well as duration. With the start of the pixellensing surveys of the center area of M31, many novae were detected as a by-product with good sampling of the outbursts which led to well-defined outburst dates and decay time scales of many novae simultaneously. In Sect. 2 we report nova detections from one of these programs. For the search of X-rays from novae in M31, we combine this nova list with novae reported in the literature. This nova list contains about 10-20 novae per year prior to the XMM-Newton and Chandra observations and may be $30 \%$ to $60 \%$ complete, while in the years before 1990 (novae to shine up during the ROSAT observations), typically less than 5 novae were reported.

M 31 (distance 780 kpc, Holland 1998; Stanek \& Garnavich 1998), with its moderate Galactic foreground absorption $\left(N_{\mathrm{H}}=6.66 \times 10^{20} \mathrm{~cm}^{-2}\right.$, Stark et al. 1992), is an ideal target to search for X-ray emission from optical novae. ROSAT has observed the full disk of the M31 galaxy (about $6.5 \mathrm{deg}^{2}$ ) twice. A ROSAT PSPC mosaic of 6 contiguous pointings with an exposure time of $25 \mathrm{ks}$ each was performed in July 1991 (first M 31 survey; Supper et al. 1997, hereafter SHP97). A second survey was made in July/August 1992 and January and July 1993 (Supper et al. 2001, hereafter SHL2001). Only one recent nova (which erupted in 1990) in M31 was reported to coincide with a catalogued ROSAT source
(Nedialkov et al. 2002). The population of SSS in M31 has been studied by Greiner et al. (1996, 2004), in particular their variability. One of the surprising results was that more fading than rising sources have been found. Coincidentally, one of these faders was the above-mentioned nova (RX J0044.0+4118; Nedialkov et al. 2002). This led to speculation that the difference in the numbers of faders and risers is due to a fraction of classical novae for which the $\mathrm{X}$-ray rising phase could be much shorter than the fading phase. Based on the, until then, known durations of the supersoft $\mathrm{X}$-ray phases, this explanation was considered unlikely. Also, the global (bulge+disk) nova rate of $\sim 37$ nova per year in M31 (Shafter \& Irby 2001), combined with the short duration of the ROSAT survey, did not suggest more than two novae among the two dozen ROSAT SSSs in M31 when taking into account the wide spread locations of the SSSs over the M31 disk. Similarly, recurrent novae were not expected to contribute to the observed SSS sample, since the outburst rate of recurrent novae in M 31 has been estimated to be only $10 \%$ of the rate of classical novae (Della Valle \& Livio 1996).

The XMM-Newton survey of M 31 has identified 856 X-ray sources (Pietsch et al. 2005, hereafter PFH2005) analyzing all observations in the XMM-Newton archive which overlap at least in part with the optical $D_{25}$ extent of the galaxy. Among them are 18 SSSs defined by $H R 1<0$ and $H R 2-E H R 2<$ -0.4 . Based on count rates in energy bands 1 to $3(0.2-0.5 \mathrm{keV}$, $0.5-1.0 \mathrm{keV}, 1.0-2.0 \mathrm{keV}), H R i$ and $E H R i$ are defined as

$H R i=\frac{B_{i+1}-B_{i}}{B_{i+1}+B_{i}} \quad$ and $\quad E H R i=2 \frac{\sqrt{\left(B_{i+1} E B_{i}\right)^{2}+\left(B_{i} E B_{i+1}\right)^{2}}}{\left(B_{i+1}+B_{i}\right)^{2}}$

for $i=1,2$, where $B_{i}$ and $E B_{i}$ denote count rates and corresponding errors in band $i$.

In addition, there are X-ray source catalogues from two deep observations of the M31 center area with Chandra ACIS S (Di Stefano et al. 2004, hereafter DKG2004) and HRC I (Kaaret 2002, hereafter K2002) sensitive to SSS emission. Several Chandra ACIS I observations (see e.g. Kong et al. 2002) are not sensitive to the detection of SSS. However, some short observations (Chandra ACIS S and HRC I) in the archive can also be searched for optical novae.

In X-rays novae may be visible as bright SSS for several years; therefore many missed novae may show up as SSS. Also recurrent novae, optically known as novae from previous or later outbursts, may show up in X-rays. For this reason and due to the uncertain outburst dates as discussed above, derived times since outburst have to be taken with care. For all M 31 novae, the distance is about the same. However, extinction within M31 may hamper the interpretation of the supersoft emission.

With the much larger emphasis on the bulge of M 31 with its high concentration of sources it was interesting to reconsider the detection rate of novae which will be presented in Sect. 3 together with a discussion of the individual objects in subsections. Finally, we discuss the results and demonstrate the wealth of information that can be expected from a continuing optical and X-ray nova survey in the center area of M 31. 


\section{M31 optical nova catalogue}

The optical novae used for cross-correlation with the $\mathrm{X}$-ray data result in part from two years of observations (June 23, 2000 to February 28, 2002) of the central part of M 31 by the continuing Wendelstein Calar Alto Pixellensing Project (WeCAPP, Riffeser et al. 2001). WeCAPP monitors a $17.2^{\prime} \times 17.2^{\prime}$ field centered on the nucleus with the $0.8 \mathrm{~m}$ telescope at Wendelstein Observatory (Germany) and the $1.23 \mathrm{~m}$ telescope at Calar Alto Observatory (Spain) continuously since 1997. The observations are carried out in $R$ and $I$ filters close to the Kron-Cousins system. The data are collected with a rather dense time coverage (up to 335 and 310 epochs in the $R$ - and $I$-bands, respectively). Data were reduced using the WeCAPP reduction pipeline mupipe, which implements an image subtraction technique (Alard \& Lupton 1998) to overcome the crowding effects and allow proper photometry of variable sources in the central bulge of M 31 (Riffeser et al. 2003). The pipeline combines the standard CCD reduction (including debiasing, flat-fielding, and filtering of cosmic ray events), position alignment, photometric calibration, and restoration of damaged pixels with full error propagation for each pixel of the CCD frame (Gössl \& Riffeser 2002). After the point spreadfunctions (PSF) of a high $S / N$ reference frame and the stacked frames are matched, the reference image is subtracted from all other frames, generating difference images for each observation night. PSF photometry of each pixel in the difference frames finally results in $4 \times 10^{6}$ pixel light curves with appropriate error bars, each of them represents the temporal variability of the flux inside the PSF centered on the particular pixel. In the full WeCAPP data set, 23770 variable sources were detected, most of them being Long Period Variables (Fliri et al. 2005). The $1 \sigma$ error radius of the astrometric solution is 0.16 . Novae are amongst the brightest variable sources in the data set. They therefore could be detected by a simple but effective algorithm. As first cut, two consecutive data points in the light curve were required that exceed a difference flux level $\Delta F_{R}$ of $6 \times 10^{-5} \mathrm{Jy}$ above the baseline (corresponding to a detection limit of $M_{R}=-2.5 \log \left(\frac{6 \times 10^{-5} \mathrm{Jy}}{F_{\text {Vega }, R}}\right) \approx 19.3$ mag with $F_{\text {Vega }, R}=3060$ Jy being the flux of Vega in the $R$-band). The light curves fulfilling this criterion are then inspected visually to extract the nova candidates. A catalogue of all 40 novae detected in the survey will be published separately. The outburst of about half the sample occured after the X-ray observations. As an example for a nova which correlates with a time variable SSS detected by XMM-Newton and Chandra, we show the optical light curve of WeCAPP-N2000-03 (Fig. 1).

We combined the WeCAPP nova list with novae from other microlensing surveys of M31: the AGAPE survey (Ansari et al. 2004), the POINT-AGAPE PACN survey (An et al. 2004; Darnley et al. 2004), the Nainital Microlensing Survey (Joshi et al. 2004) and the survey by Tomaney \& Crotts (1996, hereafter TC96). We added novae from IAU circulars and astronomical telegrams (ATEL). We included novae from the $\mathrm{H} \alpha$ searches of Shafter \& Irby (2001, hereafter SI2001), Rector et al. (1999, hereafter RJC99, nova and nova candidate

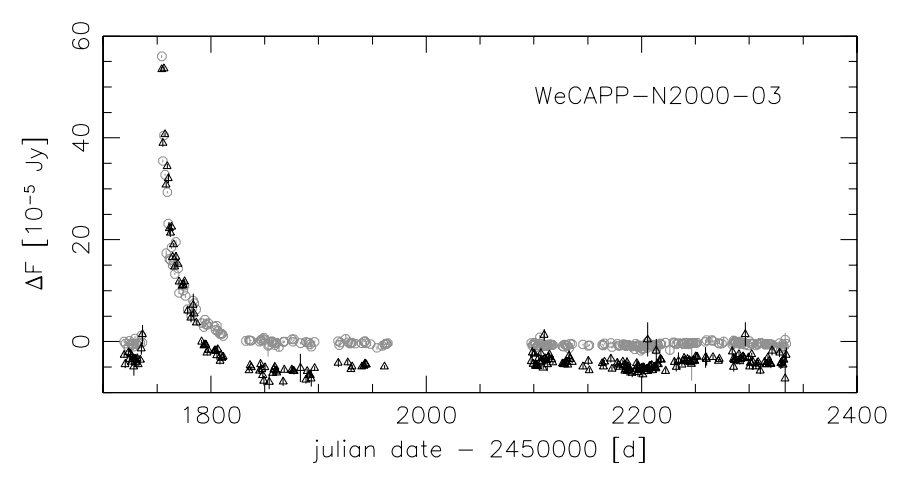

Fig. 1. Optical light curve of WeCAPP-N2000-03 in $R$ (grey circles) and $I$ (black triangles). The $I$ band data show a negative bias as the reference image contained nova epochs.

lists ${ }^{1}$ ), Ciardullo et al. (1990), and Ciardullo et al. (1987, hereafter CFN87). We added the lists by Sharov and colleagues (Sharov \& Alksnis 1991, 1992a,b; Sharov 1993, 1994; Sharov \& Alksnis 1994, 1995, 1996, 1997; Sharov et al. 1998; Sharov \& Alksnis 1998; Sharov et al. 2000). For two recurrent novae we use the naming convention provided in the General Catalogue of Variable Stars (Samus et al. 2004). We refrained from using positions of earlier nova catalogues for the crosscorrelation as nova positions in the earlier catalogues are only determined to 0.1 arcmin or worse. This might lead to many spurious correlations specifically in the central region of M31, which is crowded with novae and X-ray sources.

\section{M 31 and M 33 optical novae detected with XMM-Newton, Chandra and ROSAT}

We compared the M31 optical nova catalogue with archival and/or published XMM-Newton, Chandra, and ROSAT data.

For XMM-Newton (Jansen et al. 2001), we reanalyzed the same archival EPIC (Strüder et al. 2001; Turner et al. 2001) observations that were used by PFH2005 for the creation of the M 31 source catalogue, i.e. pointings c1 to c4 (June 2000 to January 2002) to the galaxy center, n1 to n3 (January 2002 and June 2002) to the northern disk, s1 and s2 (January 2002) to the southern disk, and h4 (January 2002) to the northwest halo (see Table 1 of PFH2005 for details). The observations were performed in the full frame mode using medium or thin filter with low background exposure times of about 10 to $50 \mathrm{ks}$. We correlated the optical nova catalogue with the sources from the PFH2005 catalogue and determined luminosities or upper limits for nova candidates for each observation. We give luminosities for the sources that are detected with at least $2 \sigma$ significance in the (0.2-1.0) keV band combining all EPIC instruments. Upper limits are $3 \sigma$ determined from the more sensitive EPIC pn camera when possible. For bright sources we analyzed the X-ray spectra.

For Chandra we correlated the optical nova catalogue with sources in the M 31 center area presented by DKG2004 and K2002 based on a $37.7 \mathrm{ks}$ ACIS S (ObsID 1575) and a 46.8 ks HRC I observation (ObsID 1912), respectively. These

\footnotetext{
1 Available at

http://www .noao.edu/outreach/rsbe/nova.html
} 
Table 1. Count rate conversion factors to unabsorbed fluxes (ECF) into the $0.2-1 \mathrm{keV}$ band for black body models with temperatures of $40 \mathrm{eV}$ and $50 \mathrm{eV}$ for different instruments and filters, including a Galactic foreground absorption of $6.66 \times 10^{20} \mathrm{~cm}^{-2}$.

\begin{tabular}{lrcr}
\hline \hline Detector & Filter & $\begin{array}{l}40 \mathrm{eV} \\
\left(10^{-11} \mathrm{erg} \mathrm{cm}^{-2} \mathrm{ct}^{-1}\right)\end{array}$ \\
\hline EPIC PN & thin & 1.77 & 1.04 \\
& medium & 2.15 & 1.22 \\
EPIC MOS1 & thin & 9.66 & 5.14 \\
& medium & 11.4 & 5.94 \\
EPIC MOS2 & thin & 9.23 & 5.00 \\
& medium & 11.1 & 5.78 \\
Chandra ACIS S & & 7.25 & 5.10 \\
Chandra HRC I & & 9.17 & 6.76 \\
ROSAT HRI & & 29.9 & 24.9 \\
ROSAT PSPC & & 6.42 & 6.55 \\
\hline
\end{tabular}

observations were performed in October 2001, between the third (c3) and forth (c4) XMM-Newton observation of the M31 center. For novae not reported in these Chandra catalogues but detected by XMM-Newton, we determined luminosities (if detected with a significance greater $2 \sigma$, else we calculated $3 \sigma$ upper limits). In addition, we searched in further short archival HRC I and ACIS S observations for nova detections and report nova correlations if more than 4 counts are detected. In only one of the many $\sim 1.2 \mathrm{ks}$ HRC I observations, a new nova candidate brighter than this limit was detected (Nova WeCAPP-N2002-01, see below). Unfortunately, upper limits also determined from these short observations do not constrain nova light curves and we therefore mostly restrain from reporting these limits. A search in the ACIS I catalogue by Kong et al. (2002) and the HRC I snap shot catalogue by Williams et al. (2004) yielded no additional nova candidates.

For ROSAT we correlated the optical nova catalogue with the source catalogues from the first and second PSPC M 31 survey (SHP97 and SHL2001) and with the HRI catalogue of Primini et al. (1993, hereafter PFJ93). In addition we searched in archival ROSAT PSPC and HRI observations for further nova correlations. From HRI information alone we cannot decide if the proposed counterpart is an SSS.

In the XMM-Newton and Chandra data, correlations with 17 optical novae in M 31 were detected. We rejected three correlations where the X-ray counterpart was classified as hard by PFH2005: the sources [PFH2005] 299, 412, and 601 which correlate with [SI2001] 1997-06, [CFN87] 26 and Nova 21 (Sharov et al. 1998), respectively. While the first two may be chance coincidences in the densely populated central area of M31, Nova 21 - which also correlates with the hard ROSAT source [SHL2001] 306 - has been classified unique from its light curve by Sharov et al. (1998) and may not be a nova at all. Five optical novae correlate with the Chandra ACIS S catalogue of DKG2004, six with the Chandra HRC I catalogue of K2002. Figure 2 demonstrates the detection of five novae near the M31 center in HRC I observation 1912. Nova

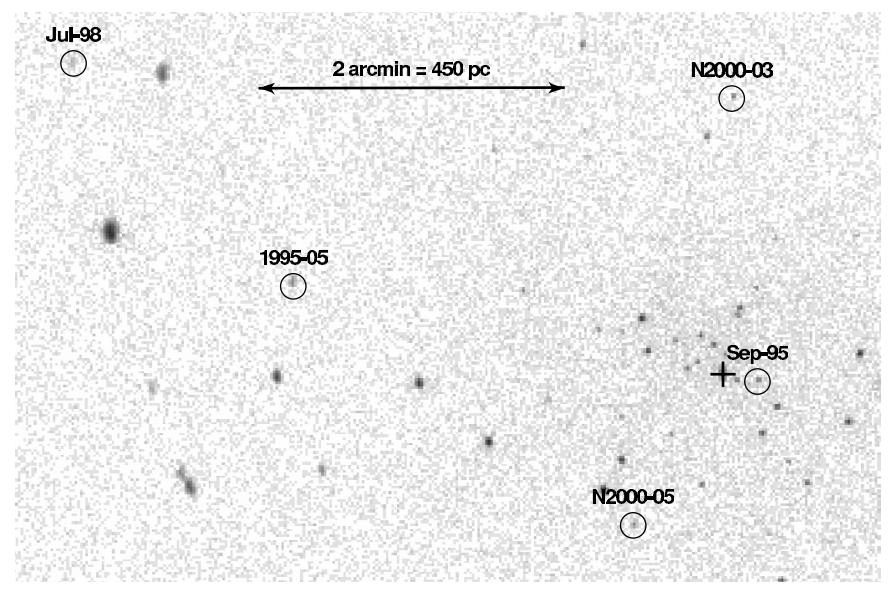

Fig. 2. Part of the Chandra HRC I image of observation 1912 used for the source catalogue of K2002. Circles with 5" radius indicate nova positions. The cross indicates the M 31 center, the aim point of the observation.

WeCAPP-N2002-01 is detected with 6 counts in the $1 \mathrm{ks}$ archival HRC I observation 2906 and [SI2001] 1997-06 with 11 counts in the $5.2 \mathrm{ks} \mathrm{HRC} \mathrm{I} \mathrm{observation} \mathrm{268.} \mathrm{Eight} \mathrm{novae} \mathrm{are}$ contained in the XMM-Newton source catalogue of PFH2005 and are detected in at least one of the contributing observations. All but one (the probable symbiotic [PFH2005] 395) XMM-Newton and Chandra HRC I nova candidates have been classified as SSS by DKG2004 and PFH2005. There are only three novae ([SI2001] 1997-06, WeCAPP-N2001-08, and WeCAPP-N2002-01) that are identified just by positional coincidence in Chandra HRC I observations and not also by their supersoft spectrum.

An et al. (2004) give correlation results of the POINTAGAPE survey list of variable stars in M 31 with 13 known novae. For four of them they propose X-ray counterparts. Novae EQ J004244+411757 and CXOM31 J004318.5+410950 are confirmed as X-ray emitting novae (novae WeCAPP-N2000-03 and WeCAPP-N2001-08). WeCAPP-N2001-12 is detected in $\mathrm{X}$-rays as described below. However, the hard X-ray transient [OBT2001] 3 (Osborne et al. 2001) is not the counterpart. The fourth X-ray candidate (CXOM31 J004222.3+411333) is more than $10^{\prime \prime}$ from the position of EQ J004242+411323 (given in Johnson et al. 1999) and about $8^{\prime \prime}$ from the position given in Osborne et al. (2001) and most probably only a chance coincidence in this crowded source region. The X-ray source is reported in the XMM-Newton catalogue as [PFH2005] 255, as a hard source that was also detected in the EINSTEIN and ROSAT HRI and PSPC surveys.

In the ROSAT PSPC and HRI data, correlations with five, respectively two, optical novae in M31 were detected. Three sources correlate with sources of the first M31 ROSAT catalogue (SHP97) and another two with sources from the second (SHL2001). The ROSAT PSPC sources have a component in the soft band indicative for SSS. The second ROSAT survey contains observations collected in three epochs. For the ROSAT nova candidates we determined luminosities for each of the epochs (SII-E1 to E3) and assume average Julian Dates (JD) of 2448840.5, 2448990.5, and 2449190.5, 
respectively. For this purpose we only merged observations of the different epochs where the source position was less than $15^{\prime}$ off-axis. For ROSAT sources from the first survey that were not detected in the second survey and vice versa, we derived upper limits when possible. The ROSAT detection and upper limits of the M 31 nova of Nedialkov et al. (2002) are also indicated. One nova correlates with a source from PFJ93, another one with two HRI observations in July 1994. Both sources are not detected in ROSAT PSPC and are only identified by positional coincidence in ROSAT HRI observations and not also by their supersoft spectrum.

Inspired by the many nova correlations in M31, we also checked the XMM-Newton and ROSAT catalogues from Pietsch et al. (2004, hereafter PMH2004) and Haberl \& Pietsch (2001, hereafter HP2001) as well as the archival Chandra data of the Local Group Sc spiral M 33 (distance $795 \mathrm{kpc}$, van den Bergh 1991) for possible nova correlations. M 33 hosts less SSS and the number of known optical novae is much less. We did not find a correlation with an optical nova within the 408 XMM-Newton sources of PMH2004. However, the ROSAT HRI source [HP2001] 93 clearly correlates with a nova. An additional M 33 nova correlation with a SSS was detected in the Chandra ACIS S observation 786.

As mentioned above, we detected three optical novae which correlate with hard sources in the PFH2005 catalogue. We consider two of them as chance coincidences. The number of hard sources in the catalog exceeds the number of SSS sources by a factor of 30. Therefore the number of chance coincidences for SSS should be smaller by the same factor. This, together with the detection of the expected X-ray spectrum and - in some cases - the expected time variability, confirms the identification of the X-ray source as optical nova counterpart. The number of sources detected only by the ROSAT HRI or Chandra HRC is much smaller than that in the PFH2005 catalogue, position errors are similar or smaller, and therefore the number of chance coincidences is even less. In addition, the time variability argument also holds for most of these sources, making all of them convincing optical nova counterparts.

We calculated intrinsic luminosities or $3 \sigma$ upper limits in the $0.2-1.0 \mathrm{keV}$ band starting from the $0.2-1 \mathrm{keV}$ count rates or upper limits in EPIC and the full count rates or upper limits in the other instruments and assuming a black body spectrum and Galactic foreground absorption. Table 1 gives energy conversion factors for the different instruments for a $40 \mathrm{eV}$ and a $50 \mathrm{eV}$ black body temperature. As one can see, the ECFs strongly change with the softness of the spectrum. Additional absorption within M31 would heavily change the observed count rate. An extrapolation to the bolometric luminosity of a nova at a given time is very uncertain; this is even more so as the temperatures of novae may vary with time after outburst and from nova to nova, and may well correspond to a spectrum even softer than $30 \mathrm{eV}$. See also the discussion of the luminosities derived from spectral modeling of a few individual optical nova candidates (Sects. 3.1, 3.9, and 3.15).

In Table 2, the results of the 23 optical nova correlations are summarized. We give nova name (or month of outburst) with optical references in Col. 1, optical position (J2000.0, Col. 2), Julian date JD of "outburst maximum" (3). We indicate if the optical maximum is well defined (to better than 5 days), or if the maximum is most likely before or after the given epoch or not well defined. As X-ray information we give the name of the source (4), distance $D$ between X-ray and optical position (5), observation number (6), JD of observation (7), days since optical nova outburst (8), X-ray luminosity in the $0.2-1.0 \mathrm{keV}$ band as described above (9), and comments like reference for detection, nova type, and SSS classification (10).

Some novae have been covered in several X-ray observations. Light curves are plotted in Fig. 3. The time variability for some of them is as expected from the ROSAT observation of V1974 Cyg. However, some others seem to brighten significantly after more than 1000 days, which we consequently interpret as recurrent novae. The individual correlations are discussed in the following subsections starting with the sources from Table 2 .

The data analysis was performed using tools in the XMM-Newton Science Analysis System (SAS) v6.1.0, EXSAS/MIDAS 03OCT_EXP/03SEPpl1.2, and FTOOLS v5.2 software packages, the imaging application DS9 v3.0b6 together with the funtools package, the mission count rate simulator WebPIMMS v3.6a and the spectral analysis software XSPEC v11.3.1.

\subsection{Nova in M 31 [SI2001] 1992-01}

This nova was reported by SI2001 from two $\mathrm{H} \alpha$ images $35 \mathrm{~d}$ apart. The positions are estimated by the authors to be accurate to $\sim 1^{\prime \prime}$. The speed class is unknown. The proposed X-ray counterpart [PFH2005] 191 is classified as SSS. The $3 \sigma \mathrm{X}$-ray positional error is $1 . ' 9$, including systematics. If we take the optical position uncertainty as $1 \sigma$, the SSS nature and the positional coincidence point at a correct identification.

The X-ray source was first detected $\sim 3300 \mathrm{~d}$ after the nova outburst during XMM-Newton observation c4. During this observation it was not in the field of view (FOV) of the EPIC pn instrument. Six days later, during observation s1 to the south of the M31 center, the source was in the FOV of all EPIC cameras, and about 240, 60, and 40 photons were collected by pn, MOS1, and MOS2, respectively. The EPIC data can best be modeled with an absorbed black body model $\left(N_{\mathrm{H}}=\left(19_{-3}^{+5}\right) \times 10^{20} \mathrm{~cm}^{-2}, k T=(48 \pm 11) \mathrm{eV}\right.$, see Fig. 4) with an unabsorbed luminosity of $6.1 \times 10^{37} \mathrm{erg} \mathrm{s}^{-1}$ in the $(0.2-1.0) \mathrm{keV}$ band. The correction factor of $\sim 50$ from absorbed to unabsorbed luminosity strongly depends on the assumed absorption column. The spectral fits show that the luminosities given in Table 2 for this source are underestimated by about a factor of five. This is caused by the higher $N_{\mathrm{H}}$ determined in the spectral fit of $\sim 20 \times 10^{20} \mathrm{~cm}^{-2}$, while for the table only Galactic foreground absorption $\left(6.66 \times 10^{20} \mathrm{~cm}^{-2}\right)$ was assumed. The nova was not detected during the three XMM-Newton observations at $2743 \mathrm{~d}$ to $3112 \mathrm{~d}$ after outburst, and it was not in the ACIS S field of Chandra observation 1575. It is covered by the Chandra HRC I observation $1912\left(\sim 13^{\prime}\right.$ offaxis), but not reported in the catalogue by K2002. We determined a $3 \sigma$ upper limit indicating that the source was not active $67 \mathrm{~d}$ before observation $\mathrm{c} 4$. 
Table 2. XMM-Newton, Chandra, and ROSAT measurements of M 31 and M 33 optical novae.

\begin{tabular}{|c|c|c|c|c|c|c|c|c|c|}
\hline \multicolumn{3}{|l|}{ Optical nova } & \multicolumn{7}{|l|}{$\mathrm{X}$-ray measurements } \\
\hline Name & $\begin{array}{l}\text { RA (h:m:s) } \\
\text { DEC (d:m:s) }\end{array}$ & $\begin{array}{c}\mathrm{JD}^{a} \\
2440000+\end{array}$ & Source name ${ }^{b}$ & $\begin{array}{l}D \\
\left({ }^{\prime \prime}\right)\end{array}$ & Observation $^{c}$ & $\begin{array}{c}\text { JD } \\
2440000+\end{array}$ & Day & $\begin{array}{c}L^{d} \\
\left(10^{36} \mathrm{erg} \mathrm{s}^{-1}\right)\end{array}$ & Comment \\
\hline $\begin{array}{l}{[\text { SI2001] }} \\
1992-01\end{array}$ & $\begin{array}{r}0: 41: 53.8 \\
41: 07: 22\end{array}$ & 8977.5 & $\begin{array}{l}\text { [PFH2005] } 191 \\
{[\mathrm{PFH} 2005] 191}\end{array}$ & $\begin{array}{l}6.4 \\
6.4\end{array}$ & $\begin{array}{r}\mathrm{c} 1 \text { (EPIC) } \\
\mathrm{c} 2 \text { (EPIC) } \\
\mathrm{c} 3(\text { EPIC) } \\
1912(\text { HRC I) } \\
\text { c4 (EPIC) } \\
\text { s1 (EPIC) }\end{array}$ & $\begin{array}{l}11720.5 \\
11906.5 \\
12089.5 \\
12213.5 \\
12280.5 \\
12286.5\end{array}$ & $\begin{array}{l}2743 \\
2929 \\
3112 \\
3236 \\
3303 \\
3309\end{array}$ & $\begin{aligned} & <1.1 \\
& <1.5 \\
& <2.2 \\
& <6.7 \\
37.0 \pm & \pm 1.7 \\
13.1 \pm 0.7 & \end{aligned}$ & SSS \\
\hline $\begin{array}{l}\text { WeCAPP- } \\
\text { N2002-01 }\end{array}$ & $\begin{array}{l}0: 42: 33.89 \\
41: 18: 23.9\end{array}$ & $12284.3^{<}$ & & $\sim 0.2$ & $\begin{array}{r}\text { c4 (EPIC) } \\
2905 \text { (HRC I) } \\
2906(\text { HRC I) }\end{array}$ & $\begin{array}{l}12280.5 \\
12290.7 \\
12427.9\end{array}$ & $\begin{array}{r}-4 \\
6 \\
144\end{array}$ & $\begin{array}{r}<0.5 \\
<9.1 \\
27.5_{-9.5}^{+12.0}\end{array}$ & \\
\hline $\begin{array}{l}\text { WeCAPP- } \\
\text { N2001-12 }\end{array}$ & $\begin{array}{l}0: 42: 34.61 \\
41: 18: 13.0\end{array}$ & 12150.9 & & $\begin{array}{l}\sim 1 \\
2.5\end{array}$ & $\begin{array}{r}\text { c3 (EPIC) } \\
1575 \text { (ACIS S) } \\
1912 \text { (HRC I) } \\
\text { c4 (EPIC) }\end{array}$ & $\begin{array}{l}12089.5 \\
12187.5 \\
12213.5 \\
12280.5\end{array}$ & $\begin{array}{r}-61 \\
37 \\
63 \\
130\end{array}$ & $\begin{aligned}<0.5 \\
<1.9 \\
0.6 \pm 0.3 \\
0.7 \pm 0.1\end{aligned}$ & $\begin{array}{l}\text { close to } \\
\text { [PFH2005] } 287 \\
\text { SSS }\end{array}$ \\
\hline [CFN87] 2 & $\begin{array}{r}0: 42: 35.0 \\
41: 13: 22\end{array}$ & 5225.0 & [PFJ93] 33 & 2.3 & $\begin{array}{l}\text { 150006h (HRI) } \\
600474 \mathrm{~h}(\mathrm{HRI}) \\
600475 \mathrm{~h}(\mathrm{HRI}) \\
400780 \mathrm{~h}(\mathrm{HRI})\end{array}$ & $\begin{array}{r}8099.5 \\
9541.5 \\
9555.5 \\
10086.5\end{array}$ & $\begin{array}{l}2874 \\
4317 \\
4331 \\
4862\end{array}$ & $\begin{array}{r}8.7 \pm 2.4 \\
<19.8 \\
<13.6 \\
<8.6\end{array}$ & \\
\hline $\begin{array}{l}{[\mathrm{SI} 2001]} \\
1990-08\end{array}$ & $\begin{array}{r}0: 42: 35.5 \\
41: 13: 48\end{array}$ & 8161.5 & [SHP97] 181 & 3.0 & $\begin{array}{r}\text { 150006h (HRI) } \\
600068 \mathrm{p} \text { (PSPC) } \\
600474 \mathrm{~h} \text { (HRI) } \\
600475 \mathrm{~h}(\mathrm{HRI})\end{array}$ & $\begin{array}{l}8099.5 \\
8465.5 \\
9541.5 \\
9555.5\end{array}$ & $\begin{array}{r}-62 \\
304 \\
1380 \\
1394\end{array}$ & $\begin{array}{r}<8.9 \\
63.5 \pm 3.7 \\
<11.7 \\
<8.1\end{array}$ & \\
\hline $\begin{array}{l}{[\text { SI2001] }} \\
1997-06\end{array}$ & $\begin{array}{l}0: 42: 40.14 \\
41: 15: 46.7\end{array}$ & 10617.5 & & $\sim 0.6$ & $\begin{array}{r}268 \text { (HRC I) } \\
1575 \text { (ACIS S) }\end{array}$ & $\begin{array}{l}11535.6 \\
12187.5\end{array}$ & $\begin{array}{r}918 \\
1570\end{array}$ & $\begin{aligned} 10.6 & \pm 3.2 \\
& <3.7\end{aligned}$ & \\
\hline AGPV 1576 & $\begin{array}{l}0: 42: 42.08 \\
41: 12: 18.0\end{array}$ & 9622.5 & $\begin{array}{l}\mathrm{J} 004242.1+411218 \\
{[\mathrm{PFH} 2005] 313}\end{array}$ & $\begin{array}{l}0.7 \\
3.1\end{array}$ & $\begin{array}{r}\text { c1 (EPIC) } \\
\text { c2 (EPIC) } \\
\text { c3 (EPIC) } \\
1912(\text { HRC I) } \\
\text { c4 (EPIC) }\end{array}$ & $\begin{array}{l}11720.5 \\
11906.5 \\
12089.5 \\
12213.5 \\
12280.5\end{array}$ & $\begin{array}{l}2098 \\
2284 \\
2467 \\
2591 \\
2658\end{array}$ & $\begin{aligned}<0.6 \\
<0.6 \\
<0.6 \\
1.2 \pm 0.4 \\
1.6 \pm 0.2\end{aligned}$ & $\begin{array}{l}\text { K2002 } \\
\text { SSS }\end{array}$ \\
\hline $\begin{array}{l}\text { RJC99 } \\
\text { Sep-95 }\end{array}$ & $\begin{array}{l}0: 42: 43.10 \\
41: 16: 04.1\end{array}$ & 9963.5 & $\begin{array}{l}\text { r1-35 } \\
\text { J004243.1+411604 }\end{array}$ & $\begin{array}{l}1.1 \\
0.2\end{array}$ & $\begin{array}{r}268 \text { (HRC I) } \\
309 \text { (ACIS S) } \\
310 \text { (ACIS S) } \\
1854 \text { (ACIS S) } \\
1575 \text { (ACIS S) } \\
1912 \text { (HRC I) } \\
2905 \text { (HRC I) }\end{array}$ & $\begin{array}{l}11535.6 \\
11697.2 \\
11728.5 \\
11923.0 \\
12187.5 \\
12213.5 \\
12290.7\end{array}$ & $\begin{array}{l}1572 \\
1734 \\
1765 \\
1960 \\
2224 \\
2250 \\
2327\end{array}$ & $\begin{array}{r}<7.0 \\
10.2 \pm 2.7 \\
16.1 \pm 3.6 \\
11.5 \pm 3.1 \\
13.4 \pm 1.2 \\
8.8 \pm 0.8 \\
22.5_{-8.5}^{+12.0}\end{array}$ & $\begin{array}{l}\text { SSS-HR, DKG2004 } \\
\text { K2002 }\end{array}$ \\
\hline $\begin{array}{l}\text { WeCAPP- } \\
\text { N2000-03 }\end{array}$ & $\begin{array}{l}0: 42: 43.97 \\
41: 17: 55.5\end{array}$ & $11753.0^{*}$ & $\begin{array}{l}{[\mathrm{PFH} 2005] 320} \\
\text { r2-60 } \\
\text { J004243.9+411755 } \\
{[\mathrm{PFH} 2005] 320}\end{array}$ & $\begin{array}{l}1.3 \\
0.8 \\
0.3 \\
1.3\end{array}$ & $\begin{array}{r}\mathrm{c} 1 \text { (EPIC) } \\
\mathrm{c} 2 \text { (EPIC) } \\
1854(\text { ACIS S) } \\
\mathrm{c} 3(\text { EPIC) } \\
1575(\text { ACIS S) } \\
1912(\text { HRC I) } \\
\text { c4 (EPIC) }\end{array}$ & $\begin{array}{l}11720.5 \\
11906.5 \\
11923.0 \\
12089.5 \\
12187.5 \\
12213.5 \\
12280.5\end{array}$ & $\begin{array}{r}-32 \\
154 \\
170 \\
337 \\
435 \\
461 \\
528\end{array}$ & $\begin{aligned}<0.7 \\
<1.4 \\
7.1 \pm 2.4 \\
9.5 \pm 0.7 \\
13.4 \pm 1.2 \\
8.4 \pm 0.8 \\
5.5 \pm 0.4\end{aligned}$ & $\begin{array}{l}\text { SSS } \\
\text { SSS-HR, DKG2004 } \\
\text { K2002 } \\
\text { SSS }\end{array}$ \\
\hline $\begin{array}{l}\text { WeCAPP- } \\
\text { N2000-05 }\end{array}$ & $\begin{array}{l}0: 42: 47.45 \\
41: 15: 07.6\end{array}$ & $11719.7^{<}$ & $\begin{array}{l}\text { r2-61 } \\
\text { J004247.4+411507 }\end{array}$ & $\begin{array}{l}1.7 \\
0.2\end{array}$ & $\begin{array}{r}310(\text { ACIS S) } \\
1854(\text { ACIS S }) \\
1570(\text { HRC I) } \\
1575(\text { ACIS S }) \\
1912(\text { HRC I) }\end{array}$ & $\begin{array}{l}11728.5 \\
11923.0 \\
12071.4 \\
12187.5 \\
12213.5\end{array}$ & $\begin{array}{r}9 \\
203 \\
352 \\
468 \\
494\end{array}$ & $\begin{array}{r}<2.2 \\
<4.2 \\
16.8_{-7.1}^{+9.7} \\
9.6 \pm 1.0 \\
5.2 \pm 0.6\end{array}$ & $\begin{array}{l}\text { SSS-HR, DKG2004 } \\
\text { K2002 }\end{array}$ \\
\hline
\end{tabular}


Table 2. continued.

\begin{tabular}{|c|c|c|c|c|c|c|c|c|}
\hline \multicolumn{3}{|l|}{ Optical nova } & \multicolumn{6}{|l|}{$\mathrm{X}$-ray measurements } \\
\hline Name & $\begin{array}{l}\text { RA (h:m:s) } \\
\text { DEC (d:m:s) }\end{array}$ & $\begin{array}{c}\mathrm{JD}^{a} \\
2440000+\end{array}$ & Source name ${ }^{b}$ & $\begin{array}{l}D \\
\left({ }^{\prime \prime}\right)\end{array}$ & Observation $^{c}$ & $\begin{array}{c}\text { JD } \\
2440000+\end{array}$ & Day & $\underset{\left(10^{36} \mathrm{erg} \mathrm{s}^{-1}\right)}{L^{d}}$ Comment \\
\hline $\begin{array}{l}{[\text { SI2001] }} \\
1997-09\end{array}$ & $\begin{array}{r}0: 42: 50.5 \\
41: 07: 48\end{array}$ & 10691.5 & [PFH2005] 347 & 2.3 & $\begin{array}{r}\text { c1 (EPIC) } \\
\text { c2 (EPIC) } \\
\text { c3 (EPIC) } \\
1912(\text { HRC I) } \\
\text { c4 (EPIC) }\end{array}$ & $\begin{array}{l}11720.5 \\
11906.5 \\
12089.5 \\
12213.5 \\
12280.5\end{array}$ & $\begin{array}{l}1029 \\
1215 \\
1398 \\
1522 \\
1589\end{array}$ & $\begin{aligned} & <1.7 \\
& <1.6 \\
& <1.2 \\
& <3.7 \\
0.7 \pm & 0.2 \mathrm{SSS}\end{aligned}$ \\
\hline $\begin{array}{l}\text { GCVS-M31- } \\
\text { V0962 }\end{array}$ & $\begin{array}{r}0: 42: 55.2 \\
41: 20: 46\end{array}$ & 10307.5 & $\begin{array}{l}\text { J004255.3+412045 } \\
{[\text { PFH2005] } 359}\end{array}$ & $\begin{array}{l}1.2 \\
1.1\end{array}$ & $\begin{array}{r}\mathrm{c} 1(\text { EPIC) } \\
\text { c2 (EPIC) } \\
\text { c3 (EPIC) } \\
1575(\text { ACIS S }) \\
1912(\text { HRC I) } \\
\text { c4 (EPIC) }\end{array}$ & $\begin{array}{l}11720.5 \\
11906.5 \\
12089.5 \\
12187.5 \\
12213.5 \\
12280.5\end{array}$ & $\begin{array}{l}1413 \\
1599 \\
1782 \\
1880 \\
1906 \\
1973\end{array}$ & $\begin{aligned} & <0.5 \text { recurrent nova } \\
& <1.1 \\
& <1.0 \\
2.2 & \pm 0.8 \\
2.9 & \pm 0.8 \mathrm{~K} 2002 \\
3.4 & \pm 0.2 \mathrm{SSS}\end{aligned}$ \\
\hline $\begin{array}{l}{[\text { SI2001] }} \\
1995-05\end{array}$ & $\begin{array}{r}0: 42: 59.3 \\
41: 16: 42\end{array}$ & 10049.5 & $\begin{array}{l}{[\mathrm{PFH} 2005] 369} \\
{[\mathrm{PFH} 2005] 369} \\
{[\mathrm{PFH} 2005] 369} \\
\text { r2-63 } \\
\text { J004259.3+411643 } \\
{[\mathrm{PFH} 2005] 369}\end{array}$ & $\begin{array}{l}2.1 \\
2.1 \\
2.1 \\
0.8 \\
1.3 \\
2.1\end{array}$ & $\begin{array}{r}400780 \mathrm{~h} \text { (HRI) } \\
268 \text { (HRC I) } \\
309 \text { (ACIS S) } \\
\mathrm{c} 1 \text { (EPIC) } \\
310 \text { (ACIS S) } \\
\mathrm{c} 2 \text { (EPIC) } \\
1854 \text { (ACIS S) } \\
\mathrm{c} 3 \text { (EPIC) } \\
1575 \text { (ACIS S) } \\
1912 \text { (HRC I) } \\
\mathrm{c} 4 \text { (EPIC) } \\
2906 \text { (HRC I) }\end{array}$ & $\begin{array}{l}10086.5 \\
11535.6 \\
11697.2 \\
11720.5 \\
11728.5 \\
11906.5 \\
11923.0 \\
12089.5 \\
12187.5 \\
12213.5 \\
12280.5 \\
12427.9\end{array}$ & $\begin{array}{r}37 \\
1486 \\
1648 \\
1671 \\
1679 \\
1857 \\
1874 \\
2040 \\
2138 \\
2164 \\
2231 \\
2378\end{array}$ & $\begin{aligned} &<5.8 \\
& 7.7 \pm 2.9 \\
& 8.9 \pm 2.6 \\
& 2.3 \pm 0.5 \mathrm{SSS} \\
& 13.8 \pm 3.3 \\
& 3.0 \pm 0.8 \mathrm{SSS} \\
& 7.1 \pm 2.4 \\
& 4.7 \pm 0.5 \mathrm{SSS} \\
& 11.4 \pm 1.0 \mathrm{SSS}-\mathrm{HR}, \mathrm{DKG} 2004 \\
& 10.5 \pm 0.9 \mathrm{~K} 2002 \\
& 7.6 \pm 0.4 \mathrm{SSS} \\
& 16.8_{-7.1}^{+9.7}\end{aligned}$ \\
\hline $\begin{array}{l}\text { [SI2001] } \\
1992-04\end{array}$ & $\begin{array}{r}0: 43: 00.7 \\
41: 14: 39\end{array}$ & 8977.5 & & $\begin{array}{l}4.1 \\
2.9\end{array}$ & $\begin{array}{r}150006 \mathrm{~h}(\mathrm{HRI}) \\
600474 \mathrm{~h}(\mathrm{HRI}) \\
600475 \mathrm{~h}(\mathrm{HRI}) \\
600674 \mathrm{~h}^{*} \text { (HRI) } \\
400780 \mathrm{~h}(\mathrm{HRI})\end{array}$ & $\begin{array}{r}8099.5 \\
9541.5 \\
9555.5 \\
9926.5 \\
10086.5\end{array}$ & $\begin{array}{r}-878 \\
564 \\
578 \\
949 \\
1109\end{array}$ & $\begin{array}{r}<9.4 \\
13.1 \pm 4.0 \\
11.8 \pm 3.3 \\
<40.0 \\
<6.3\end{array}$ \\
\hline $\begin{array}{l}\text { RJC99 } \\
\text { Jul-98 }\end{array}$ & $\begin{array}{l}0: 43: 06.96 \\
41: 18: 09.9\end{array}$ & 11019.5 & $\begin{array}{l}{[\mathrm{PFH} 2005] 395} \\
\text { r3-115 } \\
\text { J004306.9+411810 } \\
{[\mathrm{PFH} 2005] 395}\end{array}$ & $\begin{array}{l}1.9 \\
1.1 \\
0.6 \\
1.9\end{array}$ & $\begin{array}{r}\mathrm{c} 1(\text { EPIC) } \\
\text { c2 (EPIC) } \\
\text { c3 (EPIC) } \\
1575(\text { ACIS S) } \\
1912(\text { HRC I) } \\
\text { c4 (EPIC) } \\
2905(\text { HRC I) } \\
2906(\text { HRC I) }\end{array}$ & $\begin{array}{l}11720.5 \\
11906.5 \\
12089.5 \\
12187.5 \\
12213.5 \\
12280.5 \\
12290.7 \\
12427.9\end{array}$ & $\begin{array}{r}701 \\
887 \\
1070 \\
1168 \\
1194 \\
1261 \\
1271 \\
1418\end{array}$ & $\begin{aligned}<2.6 \text { symbiotic } ? \\
<4.3 \\
0.9 \pm 0.4 \text { SSS } \\
5.0 \pm 0.7 \text { SSS-HR, DKG2004 } \\
3.8 \pm 0.9 \text { K2002 } \\
20.1 \pm 0.7 \text { hard spectrum, see Fig. } 6 \\
80.0 \pm 15.0 \\
<8.4\end{aligned}$ \\
\hline $\begin{array}{l}\text { WeCAPP- } \\
\text { N2001-08 }\end{array}$ & $\begin{array}{l}0: 43: 18.61 \\
41: 09: 49.1\end{array}$ & $12094.6^{<}$ & J004318.5+410950 & 1.2 & $\begin{array}{r}\text { c3 (EPIC) } \\
1912(\text { HRC I) } \\
\text { c4 (EPIC) }\end{array}$ & $\begin{array}{l}12089.5 \\
12213.5 \\
12280.5\end{array}$ & $\begin{array}{r}-5 \\
119 \\
186\end{array}$ & $\begin{aligned} & <0.9 \\
5.3 & \pm 2.0 \mathrm{~K} 2002 \\
& <0.5\end{aligned}$ \\
\hline $\begin{array}{l}\text { GCVS-M31- } \\
\text { V1067 }\end{array}$ & $\begin{array}{l}0: 43: 28.76 \\
41: 21: 42.6\end{array}$ & 10970.5 & $\begin{array}{l}\text { [PFH2005] } 456 \\
{[\mathrm{PFH} 2005] 456}\end{array}$ & $\begin{array}{l}1.1 \\
1.1\end{array}$ & $\begin{array}{r}\text { c1 (EPIC) } \\
\text { c2 (EPIC) } \\
\text { c3 (EPIC) } \\
1912(\text { HRC I) } \\
\text { c4 (EPIC) }\end{array}$ & $\begin{array}{l}11720.5 \\
11906.5 \\
12089.5 \\
12213.5 \\
12280.5\end{array}$ & $\begin{array}{r}750 \\
936 \\
1119 \\
1243 \\
1310\end{array}$ & $\begin{aligned} & <1.0 \text { recurrent nova } \\
& <1.7 \\
1.3 & \pm 0.4 \mathrm{SSS} \\
& <5.0 \\
1.7 & \pm 0.3 \mathrm{SSS}\end{aligned}$ \\
\hline $\begin{array}{l}{[\mathrm{SI} 2001]} \\
1991-12\end{array}$ & $\begin{array}{r}0: 43: 33.5 \\
41: 16: 22\end{array}$ & 8280.5 & $\begin{array}{l}\text { [SHL2001] } 246 \\
\text { [SHL2001] } 246\end{array}$ & $\begin{array}{l}10.5 \\
10.5\end{array}$ & $\begin{array}{r}\text { 600068p (PSPC) } \\
\text { SII-E1 (PSPC) } \\
\text { SII-E2 (PSPC) } \\
\text { SII-E3 (PSPC) }\end{array}$ & $\begin{array}{l}8465.5 \\
8840.5 \\
8990.5 \\
9190.5\end{array}$ & $\begin{array}{l}185 \\
560 \\
710 \\
910\end{array}$ & $\begin{aligned}<14.8 \\
88.3 \pm 10.1 \text { strong soft component } \\
51.4 \pm 6.2 \text { strong soft component } \\
<5.8\end{aligned}$ \\
\hline $\begin{array}{l}{[\mathrm{SI} 2001]} \\
1990-16\end{array}$ & $\begin{array}{l}0: 44: 04.71 \\
41: 18: 21.7\end{array}$ & 8151.9 & [SHP97] 268 & 2.06 & $\begin{array}{r}\text { 150006h (HRI) } \\
600067 \mathrm{p}(\mathrm{PSPC}) \\
\text { SII-E1 (PSPC) } \\
\text { SII-E2 (PSPC) }\end{array}$ & $\begin{array}{l}8099.5 \\
8464.5 \\
8840.5 \\
8990.5\end{array}$ & $\begin{array}{r}-52 \\
313 \\
689 \\
839\end{array}$ & $\begin{aligned} & <28.7 \\
12.1 & \pm 1.9 \text { SSS, see Nedialkov } \\
& <8.2 \text { et al. }(2002) \\
& <2.9\end{aligned}$ \\
\hline
\end{tabular}


Table 2. continued.

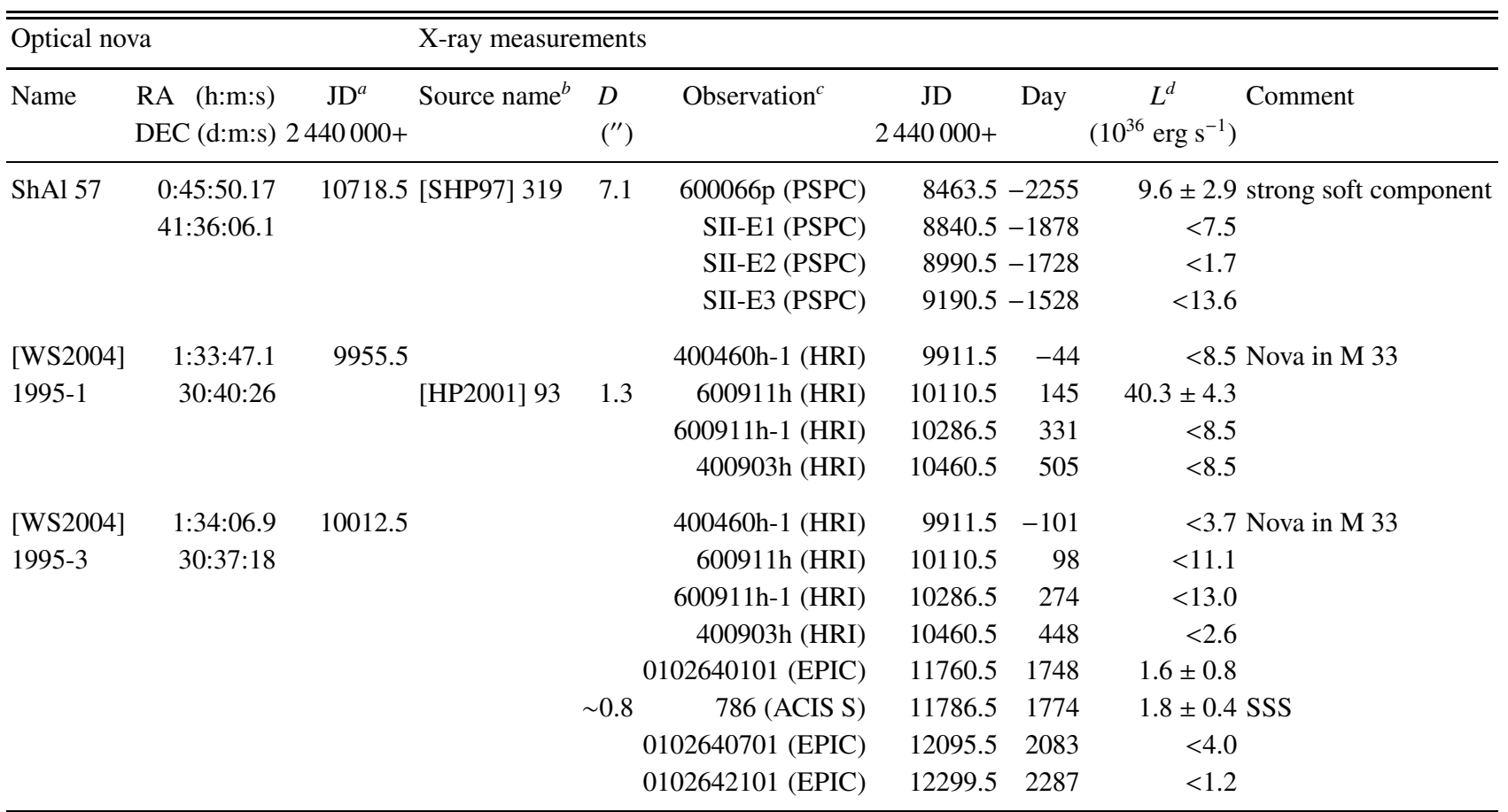

Notes: $\quad{ }^{a *}$ Indicates well defined date of optical maximum, ${ }^{<}$and ${ }^{>}$maximum before or after, else badly defined (see text).

${ }^{b}$ Full source names from K2002 are CXOM31 Jhhmmss.s+ddmmss.

${ }^{c}$ For XMM-Newton c1 corresponds to ObsID 0112570401, c2 to 0112570601, c3 to 0112570701, c4 to 0112570101 and s1 to 0112570201; for Chandra we give ObsID and camera used (see text); for the second ROSAT survey SII-E1, SII-E2, SII-E3 indicate the different epochs (see text); ${ }^{*}$ Merged with ROSAT HRI observation $600675 \mathrm{~h}$.

${ }^{d}$ Unabsorbed luminosity in $0.2-1.0 \mathrm{keV}$ band assuming a $50 \mathrm{eV}$ black body spectrum with Galactic absorption; upper limits are $3 \sigma$.

The rise of the X-ray flux of the nova rather late after the optical outburst could indicate that nova [SI2001] 1992-01 is a recurrent nova that had a new outburst after about 8 years which was not optically detected and that is responsible for the observed X-rays. Of course, we also cannot exclude that a physically different nova or even a classical SSS could be the counterpart.

\subsection{Nova in M 31 WeCAPP-N2002-01}

The time of outburst maximum of the nova WeCAPP-N200201 (also reported in IAU Circular by Fiaschi et al. 2002) can only be determined to an accuracy of 8 days, as there is an observation gap in the WeCAPP data. It is a moderately fast nova. Its outburst occurred after the XMM-Newton and Chandra observations analyzed in K2002, DKG2004, and PFH2005 where it was not detected. However, in the $1.2 \mathrm{ks}$ archival Chandra HRC I observation $2906-\sim 144 \mathrm{~d}$ after the nova outburst we find 6 photons consistent with the Chandra HRMA/HRC I point spread function at the position of the nova. The HRC I provides no spectral information. Therefore it will be important to determine from XMM-Newton EPIC or Chandra ACIS-S observations if the spectrum of the source is supersoft.

\subsection{Nova in M 31 WeCAPP-N2001-12}

The time of outburst maximum of the nova WeCAPP-N200112 (also reported in IAU Circular by Fiaschi et al. 2001) and in the POINT-AGAPE variable star catalogue (An et al. 2004) can only be determined to an accuracy of about 10 days, as there is an observation gap in the WeCAPP data of $5 \mathrm{~d}$ before and $12 \mathrm{~d}$ after the detection in the rising phase. The nova is classified as fast.

An et al. (2004) propose the hard X-ray transient [OBT2001] 3 (Osborne et al. 2001) as counterpart, which is source 287 in the PFH2005 catalogue. However, several points speak against this identification: (I) the position of this bright $\mathrm{X}$-ray source is significantly offset from the nova position by $4.4^{\prime \prime}$, (II) the X-ray source was found active $430 \mathrm{~d}$ before the nova outburst and was off in the XMM-Newton observations $244 \mathrm{~d}$ and $60 \mathrm{~d}$ before the nova outburst, (III) the $\mathrm{X}$-ray source showed a hard spectrum with a luminosity of $1.1 \times 10^{37} \mathrm{erg} \mathrm{s}^{-1}$ in the $0.3-10 \mathrm{keV}$ band. Therefore, a neutron star or black hole X-ray transient is the more likely identification for [OBT2001] 3 .

During observation c4 - $130 \mathrm{~d}$ after the nova outburst we detect a faint SSS close to the position of [PFH2005] 287, which nicely coincides within the $3 \sigma$ positional error of 3.6" with the nova position. The source was not present in observation c3 half a year earlier. Also for the Chandra ACIS S observation $157537 \mathrm{~d}$ after outburst, we can only determine an upper limit, which, however, is not very constraining. During the HRC I observation $191263 \mathrm{~d}$ after the outburst, we detected a source at the nova position with $(8.1 \pm 3.5)$ counts. It will be interesting to see if the X-ray brightness of this well-sampled fast nova further increases. 


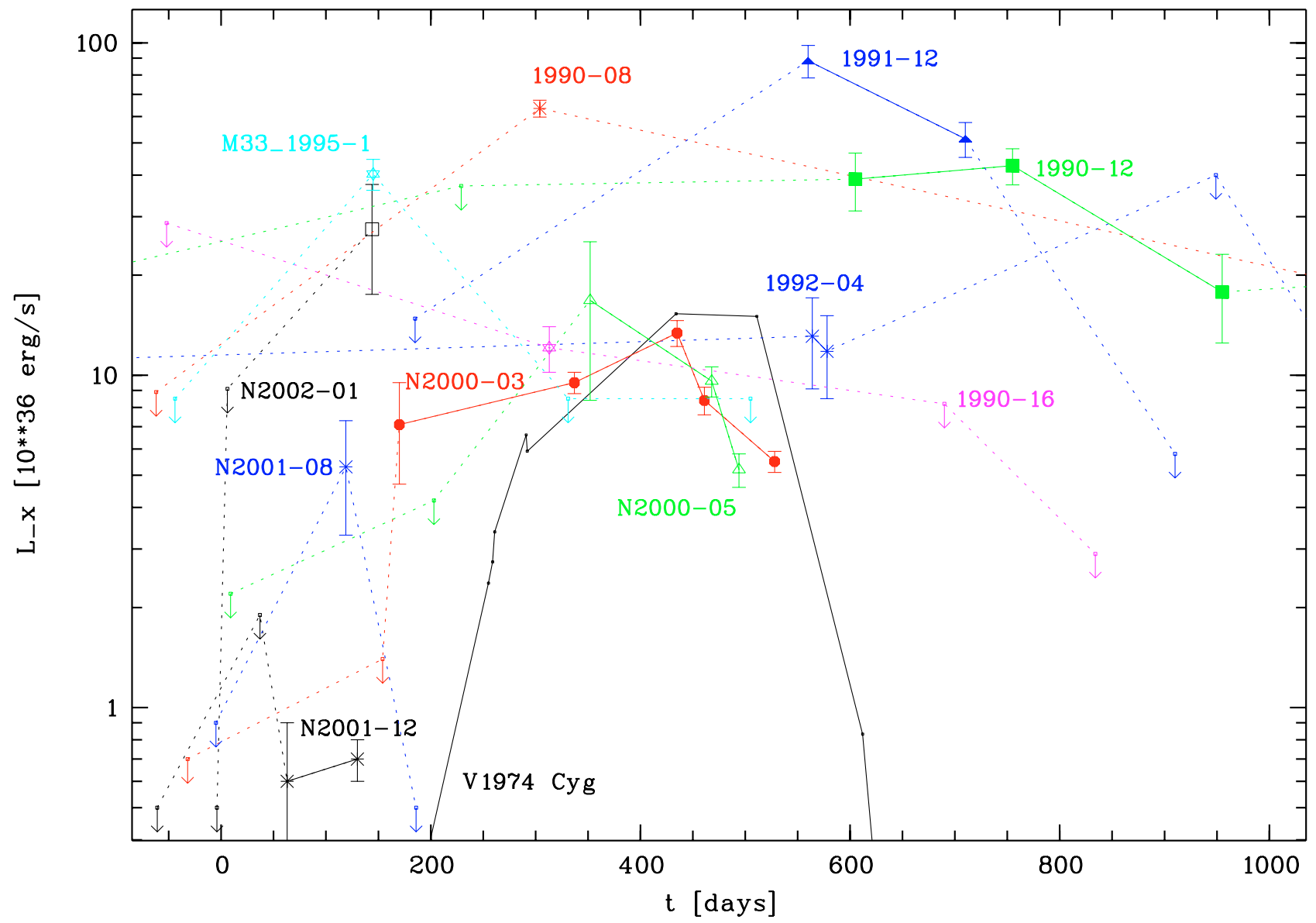

Fig. 3. Light curves for M 31 and M 33 novae that were detected within $1000 \mathrm{~d}$ after outburst. Detections of individual novae are connected by solid lines, and connections to upper limits are marked by dashed lines. The light curve from nova V1974 Cyg is adapted from Krautter et al. (1996) assuming $1 \mathrm{cts} \mathrm{s}^{-1}=0.2 \times 10^{36} \mathrm{erg} \mathrm{s}^{-1}$.

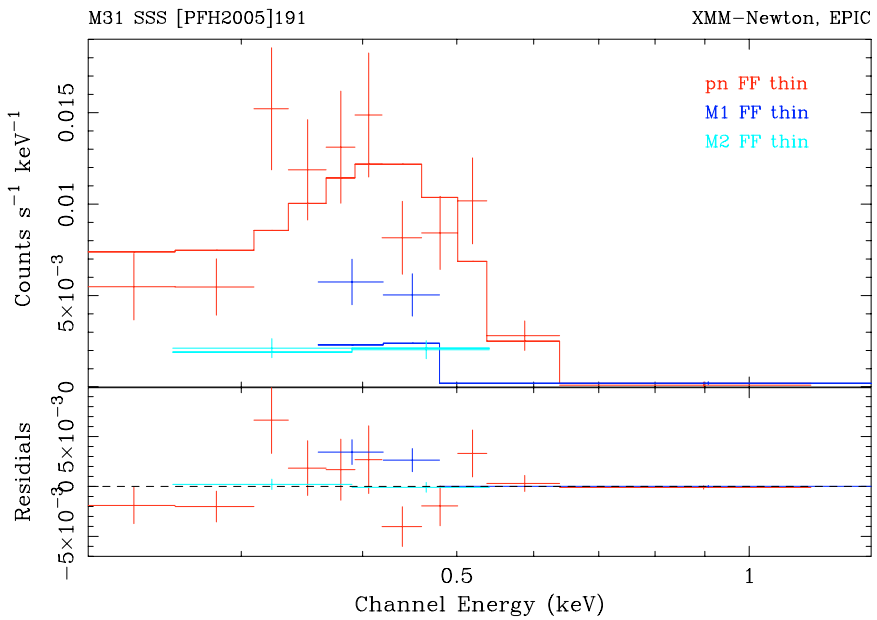

Fig. 4. XMM-Newton EPIC spectrum of source [PFH2005] 191 (Nova in M 31 [SI2001] 1992-01) for observation s1. The absorbed black body fit to the data (see Sect. 3.1) is shown in the upper panel.

\subsection{Nova in M31 [CFN87] 2}

This nova was reported by CFN87 from eight $\mathrm{H} \alpha$ images spread over $12 \mathrm{~d}$. The proposed X-ray counterpart [PFJ93] 33 closely coincides with the nova position. The source can not be resolved from nearby bright sources with the ROSAT PSPC in survey one and survey two and was no longer detected in ROSAT HRI observations more than 4 yr later. The $\mathrm{X}$-ray source was detected $7.9 \mathrm{yr}$ after the nova outburst. This could indicate that nova [CFN87] 2 is a recurrent nova which had a new outburst after about 7 years which was not optically detected and which is responsible for the observed X-rays. As only the HRI detected the source, we have no information on the X-ray spectrum.

\subsection{Nova in M31 [SI2001] 1990-08}

This nova was reported by SI2001 from two $\mathrm{H} \alpha$ images $44 \mathrm{~d}$ apart. The position is estimated by the authors to be accurate to $\sim 1^{\prime \prime}$. The proposed X-ray counterpart [SHP97] 181 has a strong soft component and the novae position is well within the $3 \sigma$ X-ray error radius. A hard source was detected in the second ROSAT survey of M31 ([SHL2001] 181) 19" offset to the NE. As this source was brighter and the sources coincided within the $3 \sigma$ error circles of 15", SHL2001 assumed that the two sources from the first and second ROSAT survey were the same with the position of [SHL2001] 181. Inspection of the ROSAT images clearly shows that the source in the second ROSAT survey is not the same source as the one detected 
in survey one. Due to the proximity of the new source in survey two, no useful upper limits can be determined during these epochs. The source was no longer active in ROSAT HRI observations $3 \mathrm{yr}$ after the PSPC detections.

\subsection{Nova in M 31 [SI2001] 1997-06}

This nova was detected by SI2001 in two $\mathrm{H} \alpha$ images $30 \mathrm{~d}$ apart. The nova is also reported by RJC99 from two images, the first already exposed $44 \mathrm{~d}$ before the first detection by SI2001. We therefore adopt nova position and date of outburst from RJC99. In X-rays, a source compatible with the nova position was detected about 2.5 years after outburst during the first Chandra HRC I observation to the center of M 31. The source is no longer detected in the Chandra ACIS S observation 1575, about $650 \mathrm{~d}$ later. The position of the source only $\sim 2$ " from the bright hard source [PFH2005] 299 prevents the detection with XMM-Newton EPIC. The Chandra HRC I provides no spectral information, and there is no ACIS S detection to identify it as a SSS.

\subsection{Nova in M 31 AGPV 1576}

This source was detected by Ansari et al. (2004) from the AGAPE project and noted as nova candidate (already end of September 1994) from the pixellensing test project by TC96. It also is a nova candidate from RJC99 (still visible in $\mathrm{H} \alpha$ in September 1995).

The source was first detected in X-rays in the Chandra HRC I observation 1912 (K2002) more than $7 \mathrm{yr}$ after outburst (only upper limit $124 \mathrm{~d}$ earlier in XMM-Newton observation c3). In the XMM-Newton observation 67 days later, the source can be classified as SSS. The novae position is well within the $3 \sigma \mathrm{X}$-ray error radii. The nova is not in the FOV of the ACIS S observation 1575.

Also in this case, the rise of the X-ray flux of the nova rather late after the optical outburst could indicate that nova AGPV 1576 is a recurrent nova that had an new outburst after about 7 years, which was not optically detected and which is responsible for the observed X-rays.

\subsection{Nova in M31 Sep-95}

This nova was reported by RJC99 from one $\mathrm{H} \alpha$ image taken September 3, 1995. Chandra observations of K2002 and DKG2004 report an X-ray source at the nova position well within the positional errors, which can be classified as SSS using ACIS S. The source position in the diffuse emission close to the M31 center near source [PFH2005] 310 prevented the detection by the XMM-Newton EPIC instruments. The source is detected beginning with the first Chandra ACIS S observations in June/July $20002.8 \mathrm{yr}$ after outburst. It is not visible in the first HRC I observation half a year earlier. However, the $3 \sigma$ upper limit derived from this $5.2 \mathrm{ks}$ observation is not much lower than the luminosity when the source is detected. After XMM-Newton observation c4, in each of the $1 \mathrm{ks}$ Chandra HRC I observations 3 to 5 counts are detected from the nova

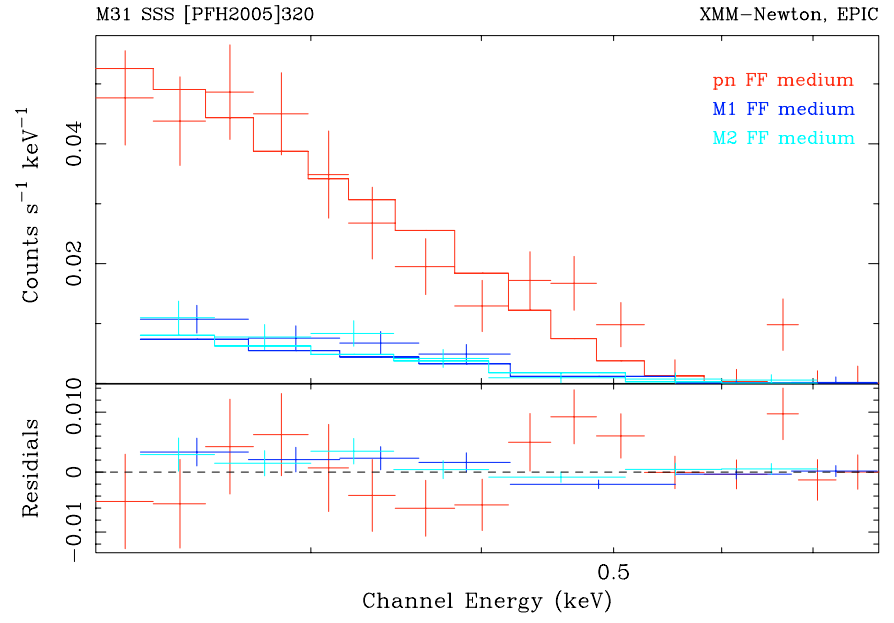

Fig. 5. XMM-Newton EPIC spectrum of source [PFH2005] 320 (nova in M31 WeCAPP-N2000-03) for observation c3. The absorbed black body fit to the data (see Sect. 3.9) is shown in the upper panel.

position indicating that the SSS was still active $3100 \mathrm{~d}$ after outburst.

\subsection{Nova in M 31 WeCAPP-N2000-03}

Nova WeCAPP-N2000-03 was detected in outburst after a $18 \mathrm{~d}$ observing gap and can be classified as a fast nova. The POINTAGAPE coverage (nova PACN-00-01, Darnley et al. 2004) of the nova outburst starts a few days after the WeCAPP light curve (see Fig. 1). Brightness estimates from Papenkova et al. (2000) found the outburst maximum about $1.5 \mathrm{~d}$ before the start of the dense WeCAPP monitoring.

In X-rays, the source ([PFH2005] 320) was first detected in the Chandra ACIS S observation $1854170 \mathrm{~d}$ after outburst. In XMM-Newton observation c2 16 days earlier, it was not detected (at least a factor of 5 fainter). It is classified as an SSS by PFH2005 and DKG2004. The source stayed bright during the following Chandra HRC I and ACIS S and the last XMM-Newton observation until at least 528 days after outburst. EPIC spectra were extracted for this SSS and simultaneously fit with a black body model with $N_{\mathrm{H}}$ fixed to the foreground value. The best fit resulted in a black body temperature of $(33 \pm 3) \mathrm{eV}$ for observation $\mathrm{c} 3$. The spectra, together with the best fit, are shown in Fig. 5. During observation $\mathrm{c} 4$ the temperature was derived to $(31 \pm 3) \mathrm{eV}$, consistent within the errors to observation $\mathrm{c} 3$. The inferred unabsorbed luminosities $(0.2-1.0 \mathrm{keV})$ during both observations are $3.1 \times 10^{37} \mathrm{erg} \mathrm{s}^{-1}$ and $2.2 \times 10^{37} \mathrm{erg} \mathrm{s}^{-1}$, respectively. The spectral fits show that the luminosities given in Table 2 for this source are underestimated by about a factor of 3.5. This is caused by the lower temperature of $\sim 30 \mathrm{eV}$, while for the table $k T=50 \mathrm{eV}$ was assumed. After XMM-Newton observation c4, in each of the $1 \mathrm{ks}$ Chandra HRC I observations 2 to 3 counts are detected from the nova position indicating that the SSS was still active $675 \mathrm{~d}$ after outburst. It will be interesting to follow the X-ray light curve of this nova with an accurately defined outburst epoch that has also been well sampled in X-rays from the start of the outburst. 


\subsection{Nova in M31 WeCAPP-N2000-05}

Nova WeCAPP-N2000-05 was detected in outburst at the beginning of the WeCAPP monitoring and showed a second maximum after about 42 days. It is probably a dwarf nova with fast decline. Chandra observations of K2002 and DKG2004 report an X-ray source at the nova position well within the positional errors which can be classified as SSS using ACIS S. Due to the bright source [PFH2005] 341 within $18^{\prime \prime}$ the nova is not resolvable by XMM-Newton EPIC. While the source is already active during Chandra observation 1570352 d after outburst, no significant emission was detected during the ACIS S observation $1854203 \mathrm{~d}$ after outburst.

\subsection{Nova in M 31 [SI2001] 1997-09}

This nova was reported by SI2001 from four $\mathrm{H} \alpha$ images $93 \mathrm{~d}$ apart. The nova is also reported by RJC99. In X-rays, a SSS was first detected compatible with the nova position during XMM-Newton observation $\mathrm{c} 4$ more than 4 years after outburst. It is not detected $67 \mathrm{~d}$ before in the Chandra HRC I observation 1912 and $191 \mathrm{~d}$ before in the XMM-Newton observation c3, respectively. Due to the rather large off-axis angle $\left(8.5^{\prime}\right)$, the Chandra upper limits are also rather large. The nova is not in the FOV of the Chandra ACIS S observation 1575.

\subsection{Nova in M 31 GCVS-M31-V0962}

This nova was reported by SI2001 as 1996-05 from one $\mathrm{H} \alpha$ image. It coincides in position with [H29] N40 (Hubble 1929) and is therefore classified as recurrent nova ( $\Delta T \sim 72 \mathrm{yr}$ or shorter). In X-rays, a source compatible with the position of the nova was reported by K2002 in HRC I observation 1912, 1906 days after outburst. In the XMM-Newton observation 67 days later it is identified with the SSS [PFH2005] 359. The source position is at the border of ACIS S3 CCD in Chandra observation 1575 and not in the source list of DKG2004. However, we determined a source luminosity during this observation, which is compatible with that of the Chandra HRC I observation $26 \mathrm{~d}$ later. In the XMM-Newton observation c3 98 d earlier and the XMM-Newton observations before the source is not detected.

\subsection{Nova in M 31 [SI2001] 1995-05}

This nova was reported by SI2001 from two $\mathrm{H} \alpha$ images $47 \mathrm{~d}$ apart. In X-rays, a source compatible with the position of the nova was detected as SSS in each of the four XMM-Newton observations to the M 31 center and in the Chandra observations of K2002 and DKG2004, 4.5 to 6.1 yr after outburst. During this time the X-ray luminosity was rising by about a factor of three. The Chandra luminosities between XMM-Newton observation c3 and c4 seem to indicate even higher source luminosity. However, it is more likely that this difference reflects ECF uncertainties. This explanation is supported by the Chandra luminosities derived from the short earlier observations starting from $4 \mathrm{yr}$ after outburst. In a ROSAT HRI observation $37 \mathrm{~d}$ after the nova outburst the source was not detected. Note that the long duration of the SSS emission in this source is only comparable to the galactic classical nova GQ Mus (Ögelman et al. 1993) or to Nova LMC 1995 (Orio et al. 2003).

\subsection{Nova in M31 [SI2001] 1992-04}

This nova was reported by SI2001 from two $\mathrm{H} \alpha$ images $35 \mathrm{~d}$ apart. The proposed X-ray counterpart was detected in two ROSAT HRI observations about $570 \mathrm{~d}$ after outburst. Due to its position close to the M31 center, it could not be resolved in the ROSAT PSPC surveys. The upper limit before the detections originates from before the nova outburst. Also the upper limits after the detection do not constrain the length of the X-ray on state. As only the HRI detected the source, we have no information on the X-ray spectrum.

\subsection{Nova in M31 Jul.-98}

This nova was reported by RJC99 from $\mathrm{H} \alpha$ images in June 1998 to July 1999. It was brightest on an H $\alpha$ image exposed on July 25, 1998. In X-rays, the source was first detected in XMM-Newton observation c3 about three years after outburst and brightened to the following Chandra HRC I and ACIS S and the XMM-Newton observation c4. In a $1.1 \mathrm{ks}$ HRC I observation $10 \mathrm{~d}$ after observation $\mathrm{c} 4$ it was brighter by another factor of 4 . In a $1.2 \mathrm{ks}$ HRC I observation $150 \mathrm{~d}$ after observation $\mathrm{c} 4$, the source was not detected and the upper limit indicates a significant decrease in brightness. The nova was not detected in XMM-Newton observation c1 and c2, $369 \mathrm{~d}$ and $183 \mathrm{~d}$ before observation c3, respectively. PFH2005 classified the source as a candidate for an X-ray binary due to its transient behavior and its hard spectrum. EPIC MOS and pn spectra extracted for observation $\mathrm{c} 4$ extend to energies above $2.5 \mathrm{keV}$ and confirm that [PFH2005] 395 during this observation is not an SSS. Simultaneous fits - with free normalization for pn as the source in this instrument is partly located on a CCD gap - yield an unacceptable reduced $\chi^{2}$ of 3.3 for 65 degrees of freedom for an absorbed black body model with a strong low-energy excess. A bremsstrahlung model with a temperature $\left(1.34_{-0.23}^{+0.29}\right) \mathrm{keV}\left(N_{\mathrm{H}}\right.$ fixed at Galactic foreground) represents the spectra best $\left(\chi^{2}=106,65\right.$ d.o.f.) among simple onecomponent models. The spectra, together with the best fit, are shown in Fig. 6. The unabsorbed luminosities inferred from the MOS spectra in the $(0.2-1.0) \mathrm{keV}$ and $(0.2-2.0) \mathrm{keV}$ bands are $5.3 \times 10^{36} \mathrm{erg} \mathrm{s}^{-1}$ and $7.8 \times 10^{36} \mathrm{erg} \mathrm{s}^{-1}$, respectively. The spectral fits show that the luminosities given in Table 2 for this source are overestimated by about a factor of four due to assuming the wrong spectral model. If the X-ray spectrum stayed the same, the flux for Chandra HRC I observation 2905 is overestimated by a factor of about nine.

During XMM-Newton observation c3, when the source was still faint, hardness ratios are typical for an SSS. Also DKG2004 classify the source as SSS based on Chandra ACIS S observation 1575 about 100 d later. Using HST images DKG2004 detected a star within the 1" error circle of the Chandra position with brightness and colors compatible with a symbiotic. This tentative identification was based on the 


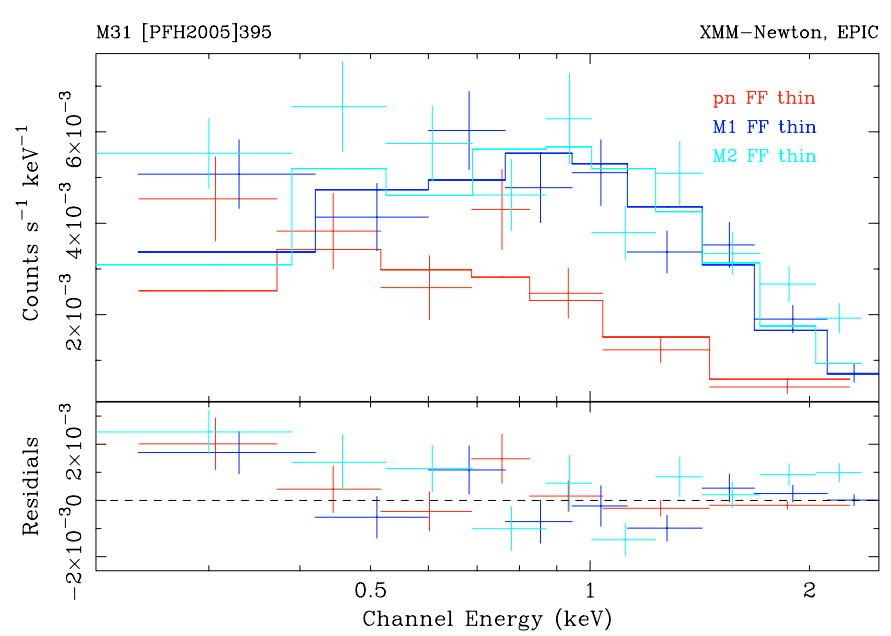

Fig. 6. XMM-Newton EPIC spectrum of source [PFH2005] 395 (nova in M 31 Jul-98) for observation c4. The absorbed bremsstrahlung fit to the data (see Sect. 3.15) is shown in the upper panel. The reduced flux in the pn spectrum is due to the location of the source on a CCD gap.

fact that several of the SSSs in the Galaxy and the Magellanic Clouds are symbiotics. However, only a few symbiotics show the very soft X-ray spectrum of a SSS with emission mainly below $0.5 \mathrm{keV}$. Another group of symbiotics exhibits X-ray spectra with the emission peaking around $0.8 \mathrm{keV}$ and might be explained by emission from optically thin plasma with temperatures in the range of a few $10^{6} \mathrm{~K}$ to a few $10^{7} \mathrm{~K}$ caused by colliding winds (Mürset et al. 1997) in the binary system. The EPIC spectra of [PFH2005] 395 are compatible with such a model which supports the identification as symbiotic. The distinct spectral change, however, from very soft $(0.5 \mathrm{keV})$ to a few $\mathrm{keV}$ spectrum on a timescale of a year has not been seen in symbiotics before, so this interpretation might not hold. An alternative might be a behaviour seen in the source 1E1339.8+2837 in M 3 (Dotani et al. 1999), which was found to switch between supersoft and hard states on timescales of 6 months. The main difference, however, are the luminosities: this source had only $10^{35} \mathrm{erg} \mathrm{s}^{-1}$ in the soft state, and about $10^{33} \mathrm{erg} \mathrm{s}^{-1}$ in the hard state. Yet another alternative are black hole transients, which also usually make soft to hard transitions, but also with luminosity changes. In the latter cases the source would not be an optical nova. The soft/hard spectral transition with correlated luminosity changes makes the source unique in our sample and may indicate that the $\mathrm{H} \alpha$ outburst of the source was incorrectly classified as an optical nova.

\subsection{Nova in M 31 [SI2001] 1990-12}

This nova was reported by SI2001 from two $\mathrm{H} \alpha$ images $45 \mathrm{~d}$ apart. The proposed X-ray counterpart [SHL2001] 230 has a strong soft component, and the novae position is well within the $3 \sigma \mathrm{X}$-ray error radius. The X-ray source was bright during SII-E1 and SII-E2 $605 \mathrm{~d}$ and $755 \mathrm{~d}$ after outburst. In SII-E3 (955 d) the luminosity decreased by a factor of two. During the first survey ( $229 \mathrm{~d})$, the source was not detected. However, the upper limit is rather high as the source was always observed at rather large off-axis angles $\left(>10^{\prime}\right)$. The source was not detected in ROSAT HRI observations before and after the PSPC observations. Especially the upper limits of the HRI observations after the PSPC detections are only mildly restraining the outburst duration.

\subsection{Nova in M 31 WeCAPP-N2001-08}

Nova WeCAPP-N2001-08 was detected in outburst after a $30 \mathrm{~d}$ observing gap and can be classified as fast nova. In $\mathrm{X}$-rays, a source compatible with the nova position was reported by K2002 from Chandra observation 2906119 d after outburst. XMM-Newton observations $124 \mathrm{~d}$ before and $67 \mathrm{~d}$ after the Chandra observation do not detect the source. The nova position is outside the FOV of ACIS S observation 1575. As only the HRC I detected the source, we have no information of the shape of the X-ray spectrum.

\subsection{Nova in M31 GCVS-M31-V1067}

This nova was reported by RJC99 from $\mathrm{H} \alpha$ images in June 1998 to July 1999. It was brightest on an H $\alpha$ image exposed on June 6, 1998. It coincides in position with [H29] N86 (Hubble 1929) and is therefore classified as recurrent nova ( $\Delta T \sim 79 \mathrm{yr}$ or shorter). In X-rays, a source compatible with the position of the nova was detected as faint SSS in the XMM-Newton observations c3 and c4 ([PFH2005] 456), $1119 \mathrm{~d}$ and $1310 \mathrm{~d}$ after outburst. In the XMM-Newton observations c1 and c2 $936 \mathrm{~d}$ and $750 \mathrm{~d}$ after the nova outburst, respectively, the source was not detected. With its distance of $\sim 8.3^{\prime}$ from the center of M31, the nova was not detected in the Chandra HRC I observation 1912 ( $3 \sigma$ upper limit well above the EPIC detection). The nova was not in the FOV in Chandra ACIS S observation 1575.

\subsection{Nova in M 31 [SI2001] 1991-12}

This nova was reported by SI2001 from one $\mathrm{H} \alpha$ image. The proposed X-ray counterpart [SHL2001] 246 has a strong soft component and the novae position is well within the $3 \sigma \mathrm{X}$-ray error radius. The X-ray source was bright during SII-E1 $560 \mathrm{~d}$ after outburst and fainter in SII-E2 (710 d). During SII-E3 (910 d) and the first survey (185 d), the source was not detected. The source was also not detected in ROSAT HRI observations before and after the outburst. We do not give HRI upper limits for this source in Table 2 as the PSPC upper limits are more constraining both in terms of luminosity and outburst duration.

\subsection{Nova in M31 [SI2001] 1990-16}

This nova was reported by SI2001 from two $\mathrm{H} \alpha$ images $2 \mathrm{~d}$ apart. The ROSAT and optical observations of the nova have been reported in detail by Nedialkov et al. (2002). However, they only give an average upper limit for the full second survey in the paper. We derived upper limits for SII-E1 and SII-E2 $689 \mathrm{~d}$ and $839 \mathrm{~d}$ after outburst, respectively. Only one observation of $1.8 \mathrm{ks}$ was available during SII-E3 which did not give a sensitive upper limit. The source was not detected during the 
ROSAT HRI observation $150006 \mathrm{~h} 52 \mathrm{~d}$ before the nova outburst. Due to the high off-axis position, the $3 \sigma$ upper limit is not very constraining.

\subsection{Nova in M 31 ShAl 57}

A special case is Nova ShAl 57 which correlates (well within the $3 \sigma$ X-ray error radius) with the source [SHP97] 319 from the first ROSAT survey, which has a strong soft component. The source vanished in ROSAT survey II. The optical outburst occurred about 6 years after the ROSAT detection (Sharov \& Alksnis 1998). However, the nova may still be the correct identification if it is a recurrent nova. Then, a previous outburst responsible for the X-ray emission might have been missed. The nova is not in the FOV of ROSAT HRI observations.

\subsection{Nova in M 33 [WS2004] 1995-1}

This nova was reported by Williams \& Shafter (2004, hereafter WS2004). In X-rays, a source compatible with the position of the nova was reported in the M33 ROSAT source catalogue of HP2001 from the combined HRI observations. We analyzed four ROSAT HRI observations that were separated by half a year with the first 44 days before the optical nova detection. Only in the observation $145 \mathrm{~d}$ after the optical nova detection do we find significant flux. Half a year later the flux dropped by at least a factor of four. As only the HRI detected the source, we have no information on the X-ray spectrum.

\subsection{Nova in M 33 [WS2004] 1995-3}

This nova was reported by WS2004. In X-rays, a source compatible with the position of the nova is clearly detected in Chandra ACIS S observation 786 about 5 years after the outburst. In this $46.3 \mathrm{ks}$ observation to the M 33 center, we detect $21.9 \pm 5.0$ and $2.9 \pm 3.5$ counts in the energy bands below $0.7 \mathrm{keV}$ and (0.7-8.0) keV, respectively, identifying the source as supersoft. The PMH2004 XMM-Newton catalogue shows no source at the position. We searched the individual observations and detected a $>2 \sigma$ excess at the source position in EPIC pn in observation 0102640101, which corresponds to a luminosity compatible with that of the Chandra observation 26 days later. The source is not in the FOV of the MOS cameras during this observation. While the upper limits from a XMM-Newton observation about one year later do not constrain the light curve, observations $6.3 \mathrm{yr}$ after the outburst seem to indicate a decline. The nova is not detected in several ROSAT HRI observations up to $1.2 \mathrm{yr}$ after the outburst. However, a source at the Chandra luminosity would have escaped detection.

\section{Discussion}

The XMM-Newton catalogue of M 31 lists 18 SSS (PFH2005). Seven coincide with optical nova positions, while another seven sources are not in the region covered by the WeCAPP survey. From the remaining four sources two ([PFH2005] 430 and 431) are very bright in at least one XMM-Newton observation and
[PFH2005] 431 shows a 865 s period in the XMM-Newton data reminiscent of a rotation period of a magnetized white dwarf (Osborne et al. 2001). These findings may classify the latter two as SSS as known from the Magellanic Clouds. These steady burning WDs in close binaries (van den Heuvel et al. 1992) differ from novae by (I) either constant or on/off X-ray emission and (II) by optical variations of at most one magnitude. The nova searches in the center area of M31 are due to lacks in the optical coverage - by far not complete and even more so in the outer disk areas. Therefore most of the remaining unidentified SSS could present X-ray emission from optical novae in the plateau phase. This view is supported by the fact that all but one (the symbiotic nova candidate RJC99 Jul.-98) of the nova correlations detected with XMM-Newton and Chandra ACIS S are classified as SSS. Also all of the ROSAT PSPC sources correlating with novae are mainly radiating in the band below $0.4 \mathrm{keV}$ and therefore SSS candidates.

The XMM-Newton catalogue of M33 lists five SSS (PMH2004). None of them coincides with a known optical nova. However, this is not surprising and may be caused just by low number statistics, and most of these SSS may still represent nova in the supersoft X-ray phase after outburst. WS2004 estimate a global nova rate for $\mathrm{M} 33$ of $2.5 \mathrm{yr}^{-1}$. However, a higher nova rate of 4.6 novae per year as derived by Della Valle et al. (1994) from a relatively frequent B monitoring of the galaxy may be more realistic (Neill \& Shara 2004). If we assume that novae from more than the past six years may show up in X-rays as indicated in our correlations in Table 2, and we take into account that the XMM-Newton M 33 survey was accumulated over 2 years, 20-40 novae with outburst dates from 1995 to end of 2002 could have been contributing. On the other hand, during this time scale only six novae are reported in the literature (see WS2004), three with outburst in 1995, one each in 1996, 1997, and 2001. In the years before the XMM-Newton observations, just one nova was reported. Therefore most if not all of the novae radiating in X-rays may have been missed in the catalogues. The detection of the two novae in M 33 with the ROSAT HRI and Chandra may reflect the denser sampling in 1995. Therefore, if one wants to detect and identify optical novae as SSS in M 33 in the future, an efficient search program for optical novae will first be necessary.

\subsection{Inference to close binary supersoft models}

We find that a major fraction of SSS as previously found with ROSAT, XMM-Newton, and Chandra are optical novae. This poses an interesting problem for the close-binary supersoft sources (CBSS), which are expected to be a numerous class of object (Di Stefano \& Rappaport 1994), with 1000 expected for M31. Since the Magellanic Clouds contain about a dozen CBSS, the new statistics of SSS in M 31, as presented here as remainders beyond the nova population, indicate that the number of SSS seemingly does not scale with the mass of the host galaxy. It thus may be worthwhile to re-consider the population studies and the detectability of CBSS. 


\subsection{Nova parameters}

The bolometric luminosity of a white dwarf envelope with hydrogen burning is directly related to the white dwarf mass (Tuchman \& Truran 1998; Sala \& Hernanz 2005a). Unfortunately, the bolometric luminosity of the SSS sources detected cannot be determined well with the present observations. Nevertheless, the evolution of the effective temperature of the source, which could be obtained only with well sampled $\mathrm{X}$-ray monitoring observations, can also constrain the parameters of the white dwarf, independent of the luminosity (as done recently for V1974 Cyg by Sala \& Hernanz 2005b).

Assuming that the material ejected by the nova explosion forms a spherical, homogeneous shell expanding at constant velocity $v$, the hydrogen mass density of the shell will evolve in time $t$ like $\rho=\frac{M_{\mathrm{H}}}{\frac{4}{3} \pi u^{3} t^{3}}$ where $M_{\mathrm{H}}$ is the ejected hydrogen mass (Krautter et al. 1996). Assuming a constant density, the column density of hydrogen will evolve with time like $N_{\mathrm{H}}\left(\mathrm{cm}^{-2}\right)=\frac{M_{\mathrm{H}}}{\frac{4}{3} \pi m_{\mathrm{H}} v^{2} t^{2}}$, where $m_{\mathrm{H}}$ is the mass of the hydrogen atom. Assuming typical values for the expansion velocity $\left(1000 \mathrm{~km} \mathrm{~s}^{-1}\right)$ and the ejected hydrogen mass $\left(10^{-5} M_{\odot}\right)$, the absorption column should reduce to $\sim 10^{21} \mathrm{~cm}^{-2}$ and thus be transparent to soft X-rays in less than one year. Indeed, for the known classical novae with soft X-ray emission, the turn on always occurred within the first year after the outburst (see for instance Orio 2004). We can use the well sampled X-ray light curves of novae WeCAPP-N2001-12 (turn on of SSS state between days 37 and 63 after outburst) and WeCAPP-N2000-03 (days 154 to 170) to estimate - under the assumptions above an ejected hydrogen mass of $\sim 10^{-6} M_{\odot}$ and $\sim 10^{-5} M_{\odot}$ in the corresponding nova outbursts, respectively.

We can compare these results with the ejected mass derived from the relationship between the hydrogen ejected mass and the rate of decline found by Della Valle et al. (2002), $\log M_{\mathrm{H}}^{\mathrm{ej}}\left(10^{-5} M_{\odot}\right)=0.274( \pm 0.197) \times \log t_{2}+0.645( \pm 0.283)$. For Nova WeCAPP-N2000-03, the outburst is almost fully covered in the optical (see discussion in Sect. 3.9), and its $t_{2}$ was $\sim 7.3$ days in $R$ and $\sim 9.7$ days in $\mathrm{I}$, which with the above relation, indicates an hydrogen ejected mass in the range (taking the error bars into account $)(3-20) \times 10^{-5} M_{\odot}$, larger than the mass derived from the X-ray light-curve. For Nova WeCAPPN2001-12, we have only an upper limit for $t_{2}$ of 11 days, leading to an upper limit for the ejected mass of $3 \times 10^{-4} M_{\odot}$. In any case, the ejected masses derived both from the X-ray and the optical light-curves are hydrogen masses, and therefore lower limits to the total ejected mass.

\subsection{X-ray detection of optical novae}

Figure 7 shows the number of optical novae per year in the optical nova catalogue of M31 used for cross-correlation with the X-ray data. X-ray detected novae are indicated separating ROSAT and XMM-Newton/Chandra detections. The time span of the M 31 observations of these satellites is also indicated. There are only few optical novae detected in the years before the ROSAT observations, which may explain the lack of nova detections before the ROSAT observations. With

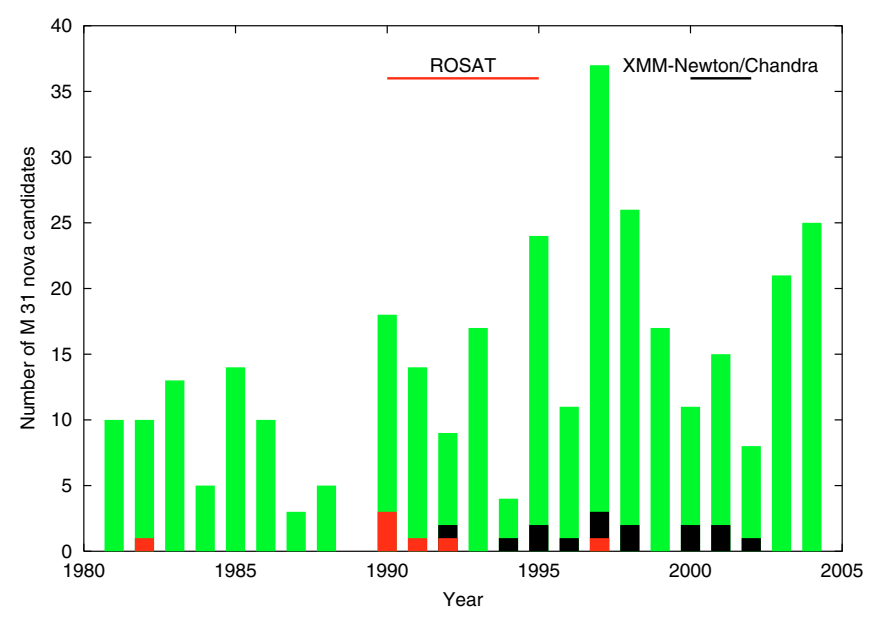

Fig. 7. Histogram of the number of optical novae per year in M31 contained in the nova catalogue used for X-ray cross-correlation (see Sect. 2). The number of optical novae showing X-ray emission is indicated coded for ROSAT and XMM-Newton/Chandra. The time span of X-ray observations is indicated.

XMM-Newton and Chandra many novae were detected which had their outburst years before the X-ray outburst. As described for the individual novae and discussed in more detail below, this may be caused due to long supersoft states of these novae or indicate that some of these novae are recurrent and had a new outburst short before the X-ray observations. Four of the 25 optical novae in 2000 and $2001(16 \%)$ are detected to turn on within a year. This percentage is only a lower limit as some novae with short supersoft states (shorter than 6 month) may have been missed in the sparse and inhomogeneous sampling of the light curves and novae in the crowded center area of M 31 are missed in XMM-Newton observations. Only prolonged sampling will allow us to decide how many additional novae will show a late turn-on as an $\mathrm{X}$-ray source.

In seven of the sources the soft X-ray emission is observed to turn on during the first year after the outburst, while in three the emission is already detected in the first X-ray observation. Eleven of the detected novae have been observed to turn off, and in five cases the SSS is still bright $5 \mathrm{yr}$ after outburst (Novae in M 31 [SI2001] 1992-01, 1995-05, AGPV 1576, RJC99 Sep.-95 and GCVS-M31-V0962). Both the large fraction of SSS detected among optical novae candidates and the long duration of the soft X-ray emission support the expected presence of a post-outburst hydrogen burning envelope left on the white dwarf.

\subsection{Supersoft $X$-ray emission from recurrent novae?}

Surprisingly enough, six of the SSS detected have turned on only between 3 and 9 years after the optically discovered nova outburst. This "delayed" appearance of the SSS is unlikely to be associated to the decrease of absorption in the ejecta.

A change in the accretion rate after the nova outburst up to the level of powering a SSS with steady hydrogen burning also seems unlikely. Kovetz et al. (1988) have shown that the irradiation of the secondary star after the nova outburst could 
cause the red dwarf to expand and induce a mass transfer rate enhanced by two orders of magnitude. They assumed that the luminosity of the irradiation source (the hydrogen burning envelope on the white dwarf) was constant and on the order of the Eddington luminosity for a certain time and that, after the irradiating source turned off, the white dwarf started to cool down. The maximum radii of the secondary star was achieved shortly $(\sim 0.1 \mathrm{yr})$ after the end of the constant luminosity phase, and then started to slowly contract, thus decreasing the accretion rate. If this process had occurred in the delayed SSS detected in M31, the irradiating white dwarf with a luminosity close to the Eddington limit would have been visible in X-rays as soon as the ejected shell became optically thin to X-rays (as mentioned above, within the first year after the outburst). But for these five cases, no SSS was detected in the first observations, performed between $\sim 700$ and $\sim 3000$ days after the outburst, depending on the source.

Finally, it is possible that these five nova candidates with delayed X-ray emission are recurrent novae, and that the optical outburst responsible for the X-ray emission was not detected. The same would be true for the nova candidate which showed $\mathrm{X}$-ray emission 6 yr before the nova outburst (ShAl 57). Two of the five delayed SSS are in fact classified as recurrent novae. If all these nova candidates are indeed recurrent novae, $29 \%$ of the novae detected as SSSs would be recurrent novae, a rate compatible with the upper limit estimated by Della Valle \& Livio (1996) for M31. Detection of delayed SSS states in $\mathrm{X}$-rays may in the future be used as a new method to classify recurrent novae in M31 and to derive the ratio of recurrent novae to classical novae.

\section{Summary and conclusions}

We searched for X-ray counterparts to optical novae detected in M31 and M33. We combined an optical nova catalogue from the WeCAPP survey with optical novae reported in the literature and correlated them with the most recent X-ray catalogues from ROSAT, XMM-Newton and Chandra, and - in addition - searched for nova correlations in archival data. We report 21 X-ray counterparts for novae in M31 (mostly SSS) and two in M33. Our sample more than triples the number of known optical novae with supersoft phase. Most of the counterparts are covered in several observations allowing us to constrain their X-ray light curves. Selected brighter sources were classified by their XMM-Newton EPIC spectra. Six counterparts are only detected in Chandra HRC I (3) or ROSAT HRI (3) observations, i.e. X-ray detectors with no energy resolution, and therefore can not be classified as supersoft.

We estimated the fraction of novae turning on as SSS within a year. The X-ray observations can be used to constrain the parameters of the white dwarf and determine the mass of the material ejected in the outburst.

More information can be expected from the analysis of already performed additional XMM-Newton and Chandra observations to the M 31 center that are not yet public. However, these observations are not homogeneously sampling the expected soft X-ray nova light curves and a dedicated observation campaign for M31 novae would be desirable. Such a campaign should cover several years in optical and X-rays and also allow to constrain the X-ray light curve of novae that are only X-ray visible for a few months.

Ongoing optical and X-ray monitoring of the central region of M31, where most of the novae are detected, should allow us to determine the length of the plateau phase of several novae and, together with nova temperature development, give a handle on the masses of the white dwarf involved. Due to the simultaneous X-ray coverage of several novae at a time and the known distance of the novae, such a program promises insights into the nova phenomenon, which is much more difficult to obtain from observation of novae in the Milky Way alone.

Acknowledgements. We thank the referee, Massimo Della Valle, for his comments, which helped to improve the manuscript considerably. Part of this work was supported by the Sonderforschungsbereich, SFB 375 of the Deutsche Forschungsgemeinschaft, DFG. The $\mathrm{XMM}$-Newton project is supported by the Bundesministerium für Bildung und Forschung / Deutsches Zentrum für Luft- und Raumfahrt (BMBF/DLR), the Max-Planck Society and the Heidenhain-Stiftung.

\section{References}

Alard, C., \& Lupton, R. H. 1998, ApJ, 503, 325

An, J. H., Evans, N. W., Hewett, P., et al. 2004, MNRAS, 351, 1071

Ansari, R., Aurière, M., Baillon, P., et al. 2004, A\&A, 421, 509

Burwitz, V., Starrfield, S., Krautter, J., \& Ness, J. 2002, in Classical Nova Explosions, ed. M. Hernanz, \& J. José, AIP Conf. Proc., 637, 377

Ciardullo, R., Ford, H. C., Neill, J. D., Jacoby, G. H., \& Shafter, A. W. 1987, ApJ, 318, 520

Ciardullo, R., Shafter, A. W., Ford, H. C., et al. 1990, ApJ, 356, 472

Darnley, M. J., Bode, M. F., Kerins, E., et al. 2004, MNRAS, 353, 571

Della Valle, M., \& Livio, M. 1996, ApJ, 473, 240

Della Valle, M., Rosino, L., Bianchini, A., \& Livio, M. 1994, A\&A, 287, 403

Della Valle, M., Pasquini, L., Daou, D., \& Williams, R. E. 2002, A\&A, 390, 155

Di Stefano, R., Kong, A. K. H., Greiner, J., et al. 2004, ApJ, 610, 247 (DKG2004)

Di Stefano, R., \& Rappaport, S. 1994, ApJ, 437, 733

Dotani, T., Asai, K., \& Greiner, J. 1999, PASJ, 51, 519

Drake, J. J., Wagner, R. M., Starrfield, S., et al. 2003, ApJ, 584, 448

Fiaschi, M., Di Mille, F., \& Cariolato, R. 2001, IAU Circ., 7709

Fiaschi, M., Di Mille, F., Cariolato, R., Swift, B., \& Li, W. D. 2002, IAU Circ., 7794

Fliri, J., Riffeser, A., Seitz, S., \& Bender, R. 2005, A\&A, submitted [arXiv: astro-ph/0507419]

Gössl, C. A., \& Riffeser, A. 2002, A\&A, 381, 1095

Gonzalez-Riestra, R., Orio, M., \& Gallagher, J. 1998, A\&AS, 129, 23

Greiner, J., Supper, R., \& Magnier, E. A. 1996, in Supersoft XRay Sources, Lecture Notes in Physics, ed. J. Greiner (Berlin Heidelberg New York: Springer), 472, 75

Greiner, J., Di Stefano, R., Kong, A., \& Primini, F. 2004, ApJ, 610, 261

Haberl, F., \& Pietsch, W. 2001, A\&A, 373, 438

Holland, S. 1998, AJ, 115, 1916

Hubble, E. P. 1929, ApJ, 69, 103

Jansen, F., Lumb, D., Altieri, B., et al. 2001, A\&A, 365, L1

Johnson, R., Modjaz, M., \& Li, W. D. 1999, IAU Circ., 7236

Joshi, Y. C., Pandey, A. K., Narasimha, D., et al. 2004, A\&A, 415, 471 
Kaaret, P. 2002, ApJ, 578, 114 (K2002)

Kato, M., \& Hachisu, I. 1994, ApJ, 437, 802

Kong, A. K. H., Garcia, M. R., Primini, F. A., et al. 2002, ApJ, 577, 738

Kovetz, A., Prialnik, D., \& Shara, M. M. 1988, ApJ, 325, 828

Krautter, J., Ögelman, H., Starrfield, S., Wichmann, R., \& Pfeffermann, E. 1996, ApJ, 456, 788

MacDonald, J., Fujimoto, M. Y., \& Truran, J. W. 1985, ApJ, 294, 263

Mürset, U., Wolff, B., \& Jordan, S. 1997, A\&A, 319, 201

Nedialkov, P., Orio, M., Birkle, K., et al. 2002, A\&A, 389, 439

Neill, J. D., \& Shara, M. M. 2004, AJ, 127, 816

Ness, J.-U., Starrfield, S., Burwitz, V., et al. 2003, ApJ, 594, L127

Ögelman, H., Beuermann, K., \& Krautter, J. 1984, ApJ, 287, L31

Ögelman, H., Orio, M., Krautter, J., \& Starrfield, S. 1993, Nature, 361, 331

Orio, M. 2004, in Rev. Mex. Astron. Astrofis. Conf. Ser., 182

Orio, M., \& Greiner, J. 1999, A\&A, 344, L13

Orio, M., \& Tepedelenlioglu, E. 2004, IAU Circ., 8435

Orio, M., Covington, J., \& Ögelman, H. 2001, A\&A, 373, 542

Orio, M., Parmar, A. N., Greiner, J., et al. 2002, MNRAS, 333, L11

Orio, M., Hartmann, W., Still, M., \& Greiner, J. 2003, ApJ, 594, 435

Osborne, J. P., Borozdin, K. N., Trudolyubov, S. P., et al. 2001, A\&A, 378, 800

Papenkova, M., Aazami, A. B., \& Li, W. D. 2000, IAU Circ., 7477

Pietsch, W., Misanovic, Z., Haberl, F., et al. 2004, A\&A, 426, 11 (PMH2004)

Pietsch, W., Freyberg, M., \& Haberl, F. 2005, A\&A, 434, 483 (PFH2005)

Primini, F. A., Forman, W., \& Jones, C. 1993, ApJ, 410, 615 (PFJ93)

Rector, T. A., Jacoby, G. H., Corbett, D. L., Denham, M., \& RBSE Nova Search Team. 1999, BAAS, 31, 1420 (RJC99)

Riffeser, A., Fliri, J., Bender, R., Seitz, S., \& Gössl, C. A. 2003, ApJ, 599, L17

Riffeser, A., Fliri, J., Gössl, C. A., et al. 2001, A\&A, 379, 362

Sala, G., \& Hernanz, M. 2005a, A\&A, 439, 1061

Sala, G., \& Hernanz, M. 2005b, A\&A, 439, 1057

Samus, N. N., Durlevich, O. V., et al. 2004, VizieR Online Data Catalog, 2250
Shafter, A. W., \& Irby, B. K. 2001, ApJ, 563, 749 (SI2001)

Sharov, A. S. 1993, Astron. Lett., 19, 230

Sharov, A. S. 1994, Astron. Lett., 20, 18

Sharov, A. S., \& Alksnis, A. 1991, Ap\&SS, 180, 273

Sharov, A. S., \& Alksnis, A. 1992a, Ap\&SS, 188, 143

Sharov, A. S., \& Alksnis, A. 1992b, Ap\&SS, 190, 119

Sharov, A. S., \& Alksnis, A. 1994, Astron. Lett., 20, 711

Sharov, A. S., \& Alksnis, A. 1995, Astron. Lett., 21, 579

Sharov, A. S., \& Alksnis, A. 1996, Astron. Lett., 22, 680

Sharov, A. S., \& Alksnis, A. 1997, Astron. Lett., 23, 540

Sharov, A. S., \& Alksnis, A. 1998, Astron. Lett., 24, 641

Sharov, A. S., Alksnis, A., Nedialkov, P. L., et al. 1998, Astron. Lett., 24,445

Sharov, A. S., Alksnis, A., Zharova, A. V., \& Shokin, Y. A. 2000, Astron. Lett., 26, 433

Shore, S. N., Starrfield, S., \& Sonneborn, G. 1996, ApJ, 463, L21

Stanek, K. Z., \& Garnavich, P. M. 1998, ApJ, 503, L131

Stark, A. A., Gammie, C. F., Wilson, R. W., et al. 1992, ApJS, 79, 77

Starrfield, S. 1989, in Classical Novae (New York: Wiley), 39

Strüder, L., Briel, U., Dennerl, K., et al. 2001, A\&A, 365, L18

Supper, R., Hasinger, G., Pietsch, W., et al. 1997, A\&A, 317, 328 (SHP97)

Supper, R., Hasinger, G., Lewin, W. H. G., et al. 2001, A\&A, 373, 63 (SHL2001)

Tomaney, A. B., \& Crotts, A. P. S. 1996, AJ, 112, 2872 (TC96)

Tuchman, Y., \& Truran, J. W. 1998, ApJ, 503, 381

Turner, M. J. L., Abbey, A., Arnaud, M., et al. 2001, A\&A, 365, L27

van den Bergh, S. 1991, PASP, 103, 609

van den Heuvel, E. P. J., Bhattacharya, D., Nomoto, K., \& Rappaport, S. A. 1992, A\&A, 262, 97

Vanlandingham, K. M., Schwarz, G. J., Shore, S. N., \& Starrfield, S. 2001, AJ, 121, 1126

Williams, B. F. M. R., Kong, A. K. H., Primini, F. A., et al. 2004, ApJ, 609, 735

Williams, S. J., \& Shafter, A. W. 2004, ApJ, 612, 867 (WS2004) 